\title{
Stability of Materials in High Temperature Water Vapor: SOFC Applications
}

\author{
E.J. Opila and N.S. Jacobson \\ NASA Glenn Research Center
}

Solid oxide fuel cell material systems require long term stability in environments containing high- temperature water vapor. Many materials in fuel cell systems react with high-temperature water vapor to form volatile hydroxides which can degrade cell performance. In this paper, experimental methods to characterize these volatility reactions including the transpiration technique, thermogravimetric analysis, and high pressure mass spectrometry are reviewed. Experimentally determined data for chromia, silica, and alumina volatility are presented. In addition, data from the literature for the stability of other materials important in fuel cell systems are reviewed. Finally, methods for predicting material recession due to volatilization reactions are described. 


\title{
Stability of Materials in High Temperature Water Vapor: SOFC Applications
}

\author{
E.J Opila and N.S. Jacobson \\ 34th International Conference on Advanced Ceramics \\ and Composites \\ Daytona Beach, FL \\ January 27, 2010
}




\section{Motivation}

- Materials in SOFC applications require stability for 10,000 to $100,000 \mathrm{~h}$ for desired cell operating performance

- High temperature water vapor is present on both anode and cathode sides of the cell

- Many gaseous metal hydroxide species are thermodynamically stable

- Formation of gaseous metal hydroxides can lead to consumption of thin layers of material and/or transport of metal species to other locations - poisoning cell operation

- Thermodynamic data for gaseous metal hydroxide stability are needed to predict cell degradation 


\section{Outline}

- Thermodynamics of gaseous metal hydroxide formation

- Experimental techniques for determination of thermodynamic data of gaseous metal hydroxide formation

- NASA GRC experimental determination of thermodynamic stability for $\mathrm{Cr}_{2} \mathrm{O}_{3}, \mathrm{SiO}_{2}$, and $\mathrm{Al}_{2} \mathrm{O}_{3}$ in high temperature water vapor

- Literature review and prediction of thermodynamic stability of $\mathrm{Ni}, \mathrm{CoO}, \mathrm{SrO}, \mathrm{CaO}, \mathrm{La}_{2} \mathrm{O}_{3}, \mathrm{MnO}, \mathrm{Pd}, \mathrm{Pt}$

- Kinetics of volatilization limited by transport through laminar gaseous boundary layer 


\section{Thermodynamics of Gaseous Metal Hydroxide Formation}

Generic reaction:

$\mathrm{MO}_{\mathrm{x}}+\mathrm{nH}_{2} \mathrm{O}(\mathrm{g})+\mathrm{mO}_{2}(\mathrm{~g})+\mathrm{qH}_{2}(\mathrm{~g})=\mathrm{MO}_{(\mathrm{x}+\mathrm{n}+2 \mathrm{~m})} \mathrm{H}_{2(\mathrm{n}+\mathrm{q})}(\mathrm{g})$

Model SOFC environments

Anode side: $\mathrm{H}_{2} \mathrm{O}(\mathrm{g})+\mathrm{H}_{2}(\mathrm{~g})$

Cathode side: $\mathrm{H}_{2} \mathrm{O}(\mathrm{g})+\mathrm{O}_{2}(\mathrm{~g})$

Pressure dependence: $\quad P_{M O H}=K_{e q} P_{H_{2} \mathrm{O}}^{n} P_{\mathrm{O}_{2}}^{m} P_{\mathrm{H}_{2}}^{q}$

$\mathrm{SiO}_{2}+2 \mathrm{H}_{2} \mathrm{O}(\mathrm{g})=\mathrm{Si}(\mathrm{OH})_{4}(\mathrm{~g})$

$1 / 2 \mathrm{Cr}_{2} \mathrm{O}_{3}+\mathrm{H}_{2} \mathrm{O}(\mathrm{g})+3 / 4 \mathrm{O}_{2}(\mathrm{~g})=\mathrm{CrO}_{2}(\mathrm{OH})_{2}(\mathrm{~g})$

$\mathrm{Ni}+2 \mathrm{H}_{2} \mathrm{O}(\mathrm{g})=\mathrm{Ni}(\mathrm{OH})_{2}(\mathrm{~g})+\mathrm{H}_{2}(\mathrm{~g})$

\begin{tabular}{|l|c|c|c|}
\hline $\mathrm{MOH}(\mathrm{g})$ & n & m & q \\
\hline $\mathrm{Si}(\mathrm{OH})_{4}$ & 2 & 0 & 0 \\
\hline $\mathrm{CrO}_{2}(\mathrm{OH})_{2}$ & 1 & $3 / 4$ & 0 \\
\hline $\mathrm{Ni}(\mathrm{OH})_{2}$ & 2 & 0 & -1 \\
\hline
\end{tabular}

Temperature dependence: $K_{\text {eq }}=\exp \left(-\frac{\Delta G}{R T}\right)=\exp \left(-\frac{\Delta H-T \Delta S}{R T}\right)$ 
Experimental techniques for determination of thermodynamic data of gaseous metal hydroxide formation

- Mass spectrometry

- Knudsen Effusion Mass Spectrometry (KEMS)

- High Pressure Free Jet Expansion MS

- Transpiration

- Thermogravimetric Analysis (TGA)

- Flame Spectroscopy 


\section{Knudsen Effusion Mass Spectrometry (KEMS)}

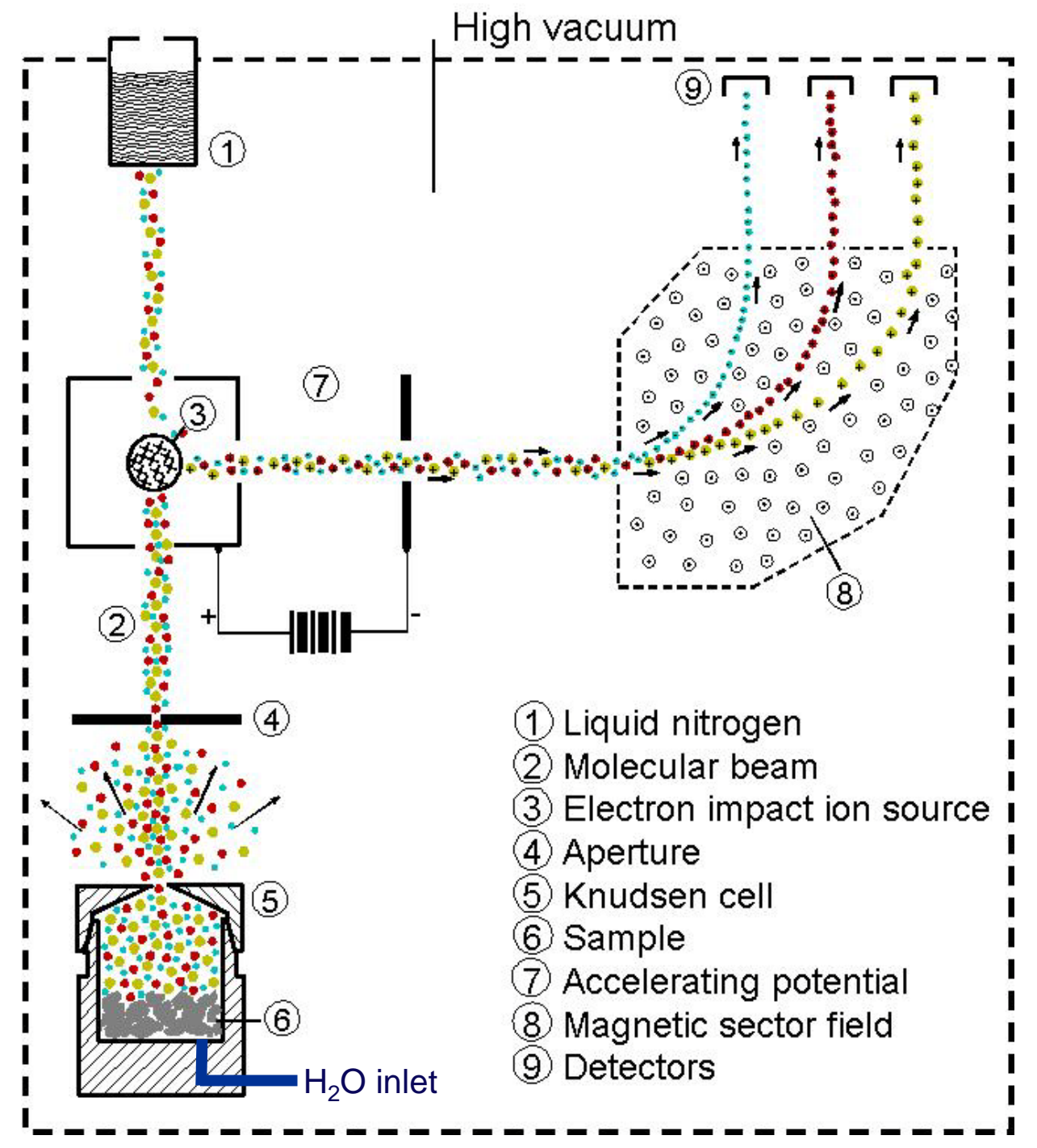

http://www.fz-juelich.de/ief/ief-2/datapool/page/227/KEMSschematisch.jpg
- Magnetic sector analysis accurate data

- High vacuum process

- Low water vapor partial pressures $\left(<10^{-5} \mathrm{bar}\right)$, therefore not representative of SOFC conditions

- Data available in literature:

$\mathrm{Si}-\mathrm{O}-\mathrm{H}(\mathrm{g})$

D. L. Hildenbrand, K. H. Lau, J. Chem. Phys., 101 [7] 6076 (1994).

$\mathrm{Mn}-\mathrm{O}-\mathrm{H}(\mathrm{g})$

D.L. Hildenbrand, K.H. Lau, J. Chem. Phys. 100 [11] 8377 (1994).

\section{$\mathrm{Pd}-\mathrm{O}(\mathrm{g})$}

J.H. Norman, H.G. Staley, W.E. Bell, J. Phys. Chem. 69 [4] 1373 (1965).

D.L. Hildenbrand, K.H. Lau, Chem. Phys. Lett. 319, 95 (2000). 


\section{High Pressure Free Jet Expansion Mass Spectrometry (HPMS)}
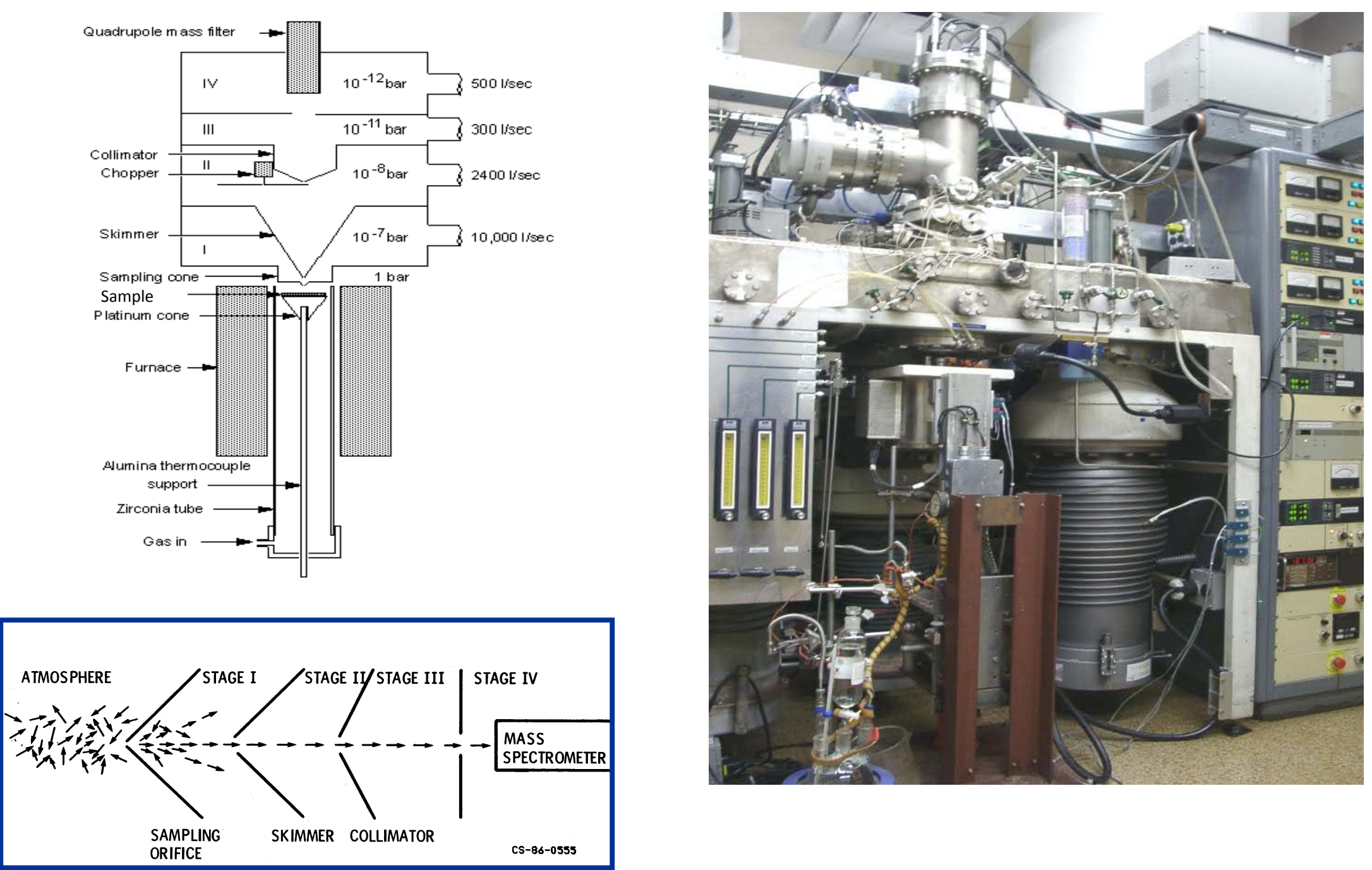


\section{High Pressure Free Jet Expansion Mass Spectrometry (HPMS)}

- Quadrupole analysis quantitative data difficult

- 1 bar sampling system

- Water vapor partial pressures possible near 1 bar representative of SOFC conditions

- Data available in literature: Si-O-H(g)

E.J. Opila, D.S. Fox, N.S. Jacobson, J. Am. Ceram. Soc. 80 [4] 1009 (1997).

$\mathrm{Cr}-\mathrm{O}-\mathrm{H}(\mathrm{g})$

G.C. Fryburg, R.A. Miller, F.J. Kohl, C.A. Stearns,

J. Electrochem. Soc.124 [11] 1738 (1977).

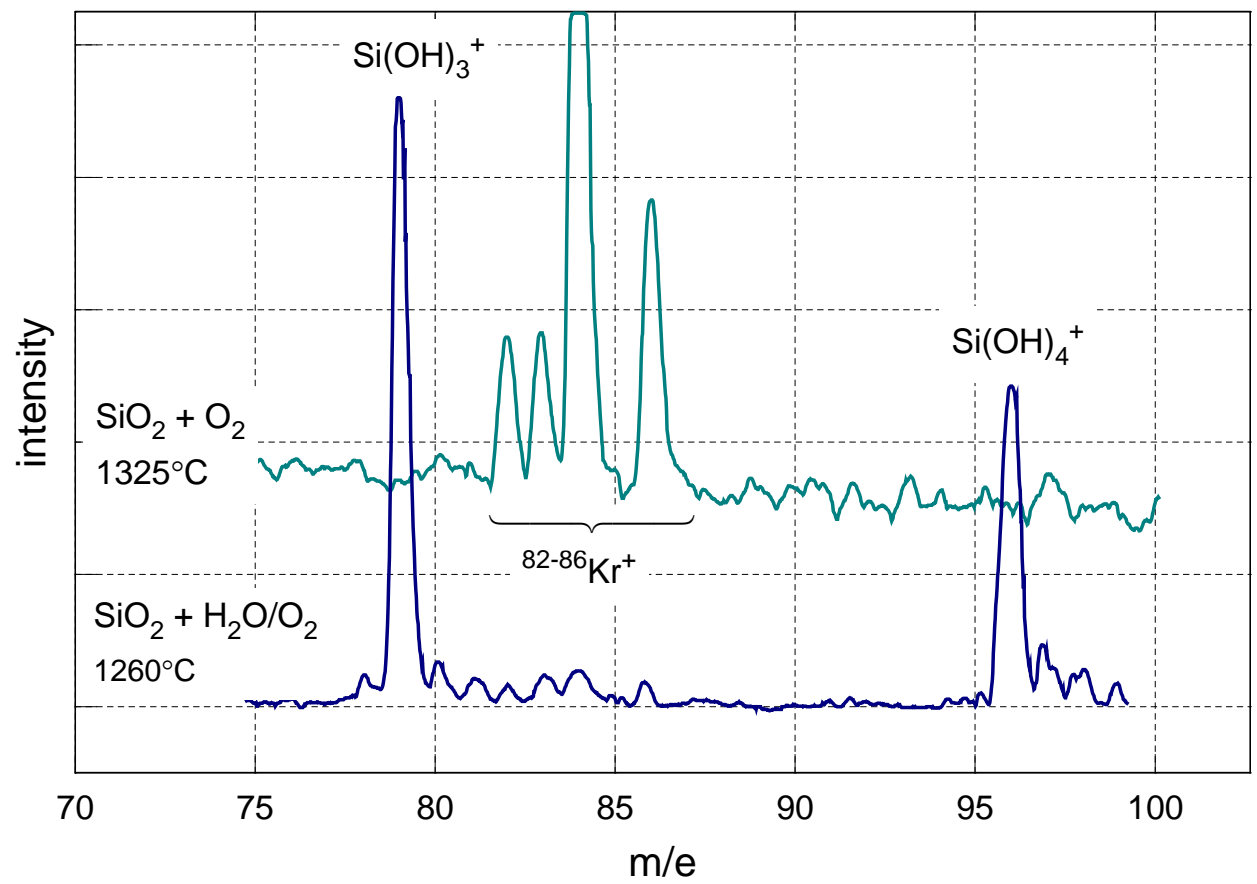

Free jet sampling mass spectrometric identification of $\mathrm{Si}(\mathrm{OH})_{4}(\mathrm{~g})$ from the reaction of $\mathrm{SiO}_{2}+\mathrm{H}_{2} \mathrm{O}(\mathrm{g})$ 


\section{The Transpiration Technique}
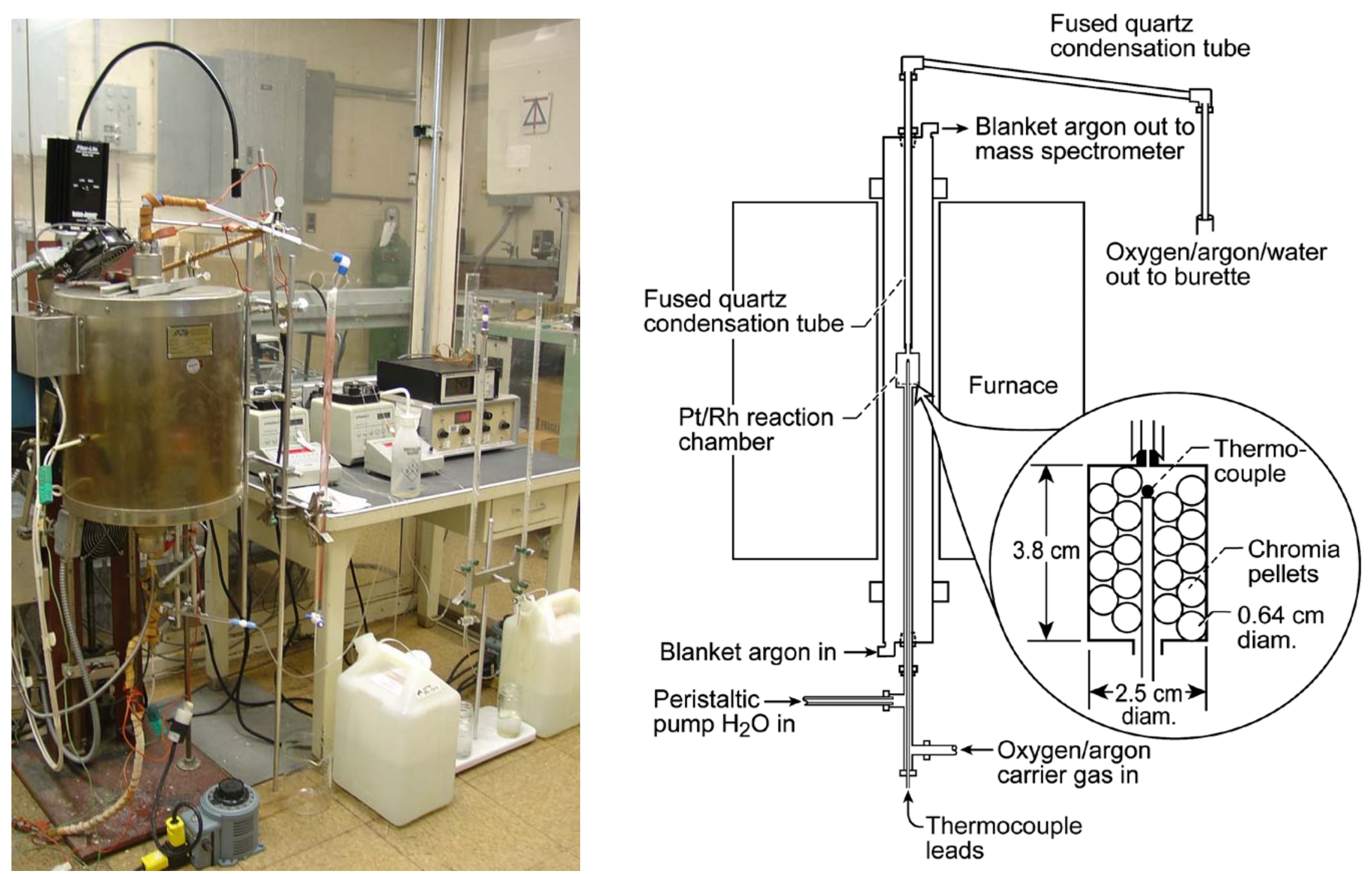


\section{The Transpiration Technique}

- Volatile species identified indirectly from pressure dependence

- Accurate pressure and temperature dependence of volatilization reaction is possible

- Water vapor partial pressures 0.1 to 1 bar - representative of SOFC conditions

- Data available in literature:

$\mathrm{Ni}-\mathrm{O}-\mathrm{H}(\mathrm{g})$ and $\mathrm{Co}-\mathrm{O}-\mathrm{H}(\mathrm{g})$

G.R. Belton, A.S. Jordan, J. Phys. Chem. 71 [12] 4114 (1967).

$\mathrm{Si}-\mathrm{O}-\mathrm{H}(\mathrm{g})$

A. Hashimoto, Geochim. Cosmo. Acta 56, 511-532 (1992).

N.S. Jacobson, E.J. Opila, D. Myers, E. CoplandJ. Chem. Thermo. 37, 1130 (2005).

$\mathrm{Ca}-\mathrm{O}-\mathrm{H}(\mathrm{g})$

K. Matsumoto, T. Sata, Bull. Chem. Soc. Jpn. 54 [3] 674 (1981).

A. Hashimoto, Geochim. Cosmo. Acta 56, 511-532 (1992).

$\mathrm{Cr}-\mathrm{O}-\mathrm{H}(\mathrm{g})$

E.J. Opila, D.L. Myers, N.S. Jacobson, I.B. Nielsen, D.F. Johnson, J.K. Olminsky, M.D. Allendorf, J. Phys. Chem. A. 111, 1971 (2007).

M. Stanislowski, E. Wessel, K. Hilpert, T. Markus, L. Singheiser, J. Electrochem. Soc. 154 [4] A295- (2007). 


\section{Thermogravimetric Analysis (TGA)}

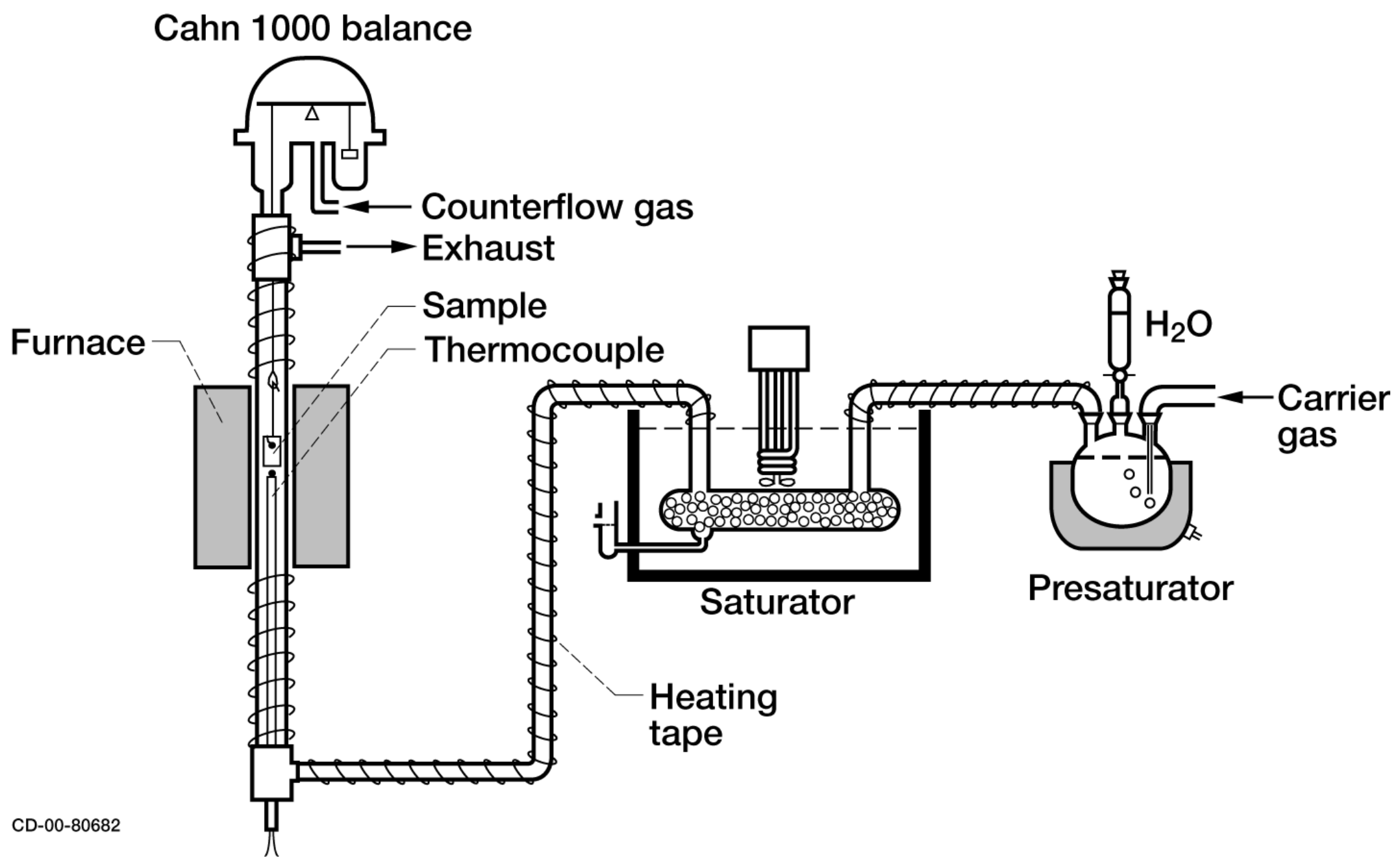




\section{Thermogravimetric analysis}

- Gas boundary layer characteristics affect volatilization rate

- Must have well defined gas flow over sample

- Equilibrium partial pressures of gaseous metal hydroxide calculated from weight loss assuming volatilization is limited by gaseous transport through laminar gas boundary layer

- Volatile species identified indirectly from pressure dependence

- Accurate pressure and temperature dependence of volatilization reaction is possible

- Water vapor partial pressures 0.1 to 1 bar - representative of SOFC conditions

- Data available in literature:

$\mathrm{Al}-\mathrm{O}-\mathrm{H}(\mathrm{g})$

E.J. Opila and D.L. Myers, J. Am. Ceram. Soc. 87 [9] 1701 (2004). 


\section{The Cr-O-H system}

- $\mathrm{Cr}_{2} \mathrm{O}_{3}$ in SOFC

- Oxide thermally grown on low temperature interconnects

- Component of $\mathrm{La}_{1-\mathrm{x}} \mathrm{M}_{\mathrm{x}} \mathrm{CrO}_{3}$ interconnect

- Many $\mathrm{Cr}-\mathrm{O}-\mathrm{H}(\mathrm{g})$ vapor species

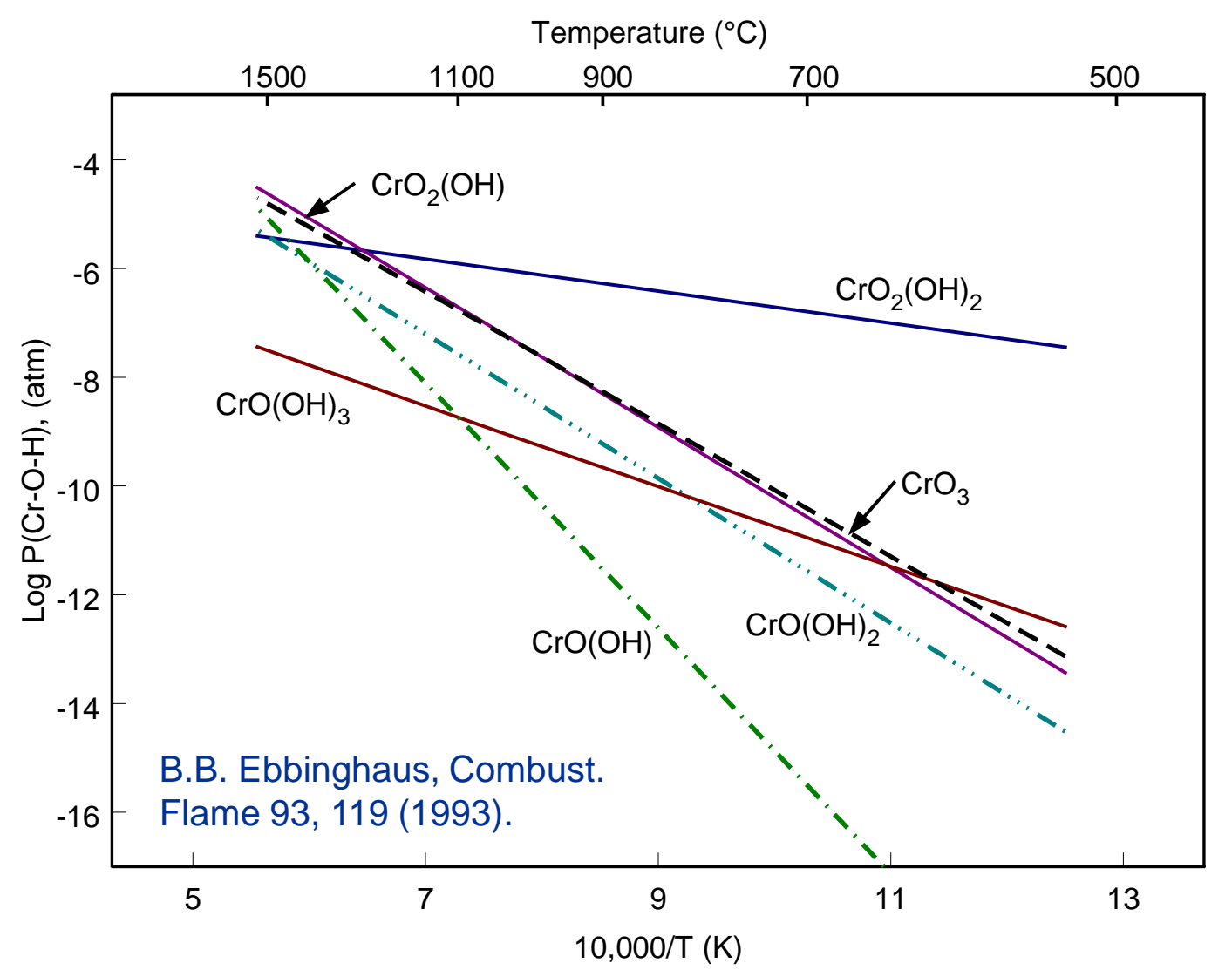




\section{The Cr-O-H system}

- Primary vapor species in $\mathrm{H}_{2} \mathrm{O}+\mathrm{O}_{2}$ environment

$$
\begin{aligned}
& 1 / 2 \mathrm{Cr}_{2} \mathrm{O}_{3}(\mathrm{~s})+3 / 4 \mathrm{O}_{2}(\mathrm{~g})=\mathrm{CrO}_{3}(\mathrm{~g}) \\
& 1 / 2 \mathrm{Cr}_{2} \mathrm{O}_{3}(\mathrm{~s})+\mathrm{H}_{2} \mathrm{O}(\mathrm{g})+3 / 4 \mathrm{O}_{2}(\mathrm{~g})=\mathrm{CrO}_{2}(\mathrm{OH})_{2}(\mathrm{~g}) \\
& 1 / 2 \mathrm{Cr}_{2} \mathrm{O}_{3}(\mathrm{~s})+1 / 2 \mathrm{H}_{2} \mathrm{O}(\mathrm{g})+1 / 2 \mathrm{O}_{2}(\mathrm{~g})=\mathrm{CrO}_{2}(\mathrm{OH})(\mathrm{g})
\end{aligned}
$$

- Confirmation of volatile species identity from pressure dependent transpiration experiment at $600^{\circ} \mathrm{C}$

mole fraction $\mathrm{H}_{2} \mathrm{O}$ or $\mathrm{O}_{2}$

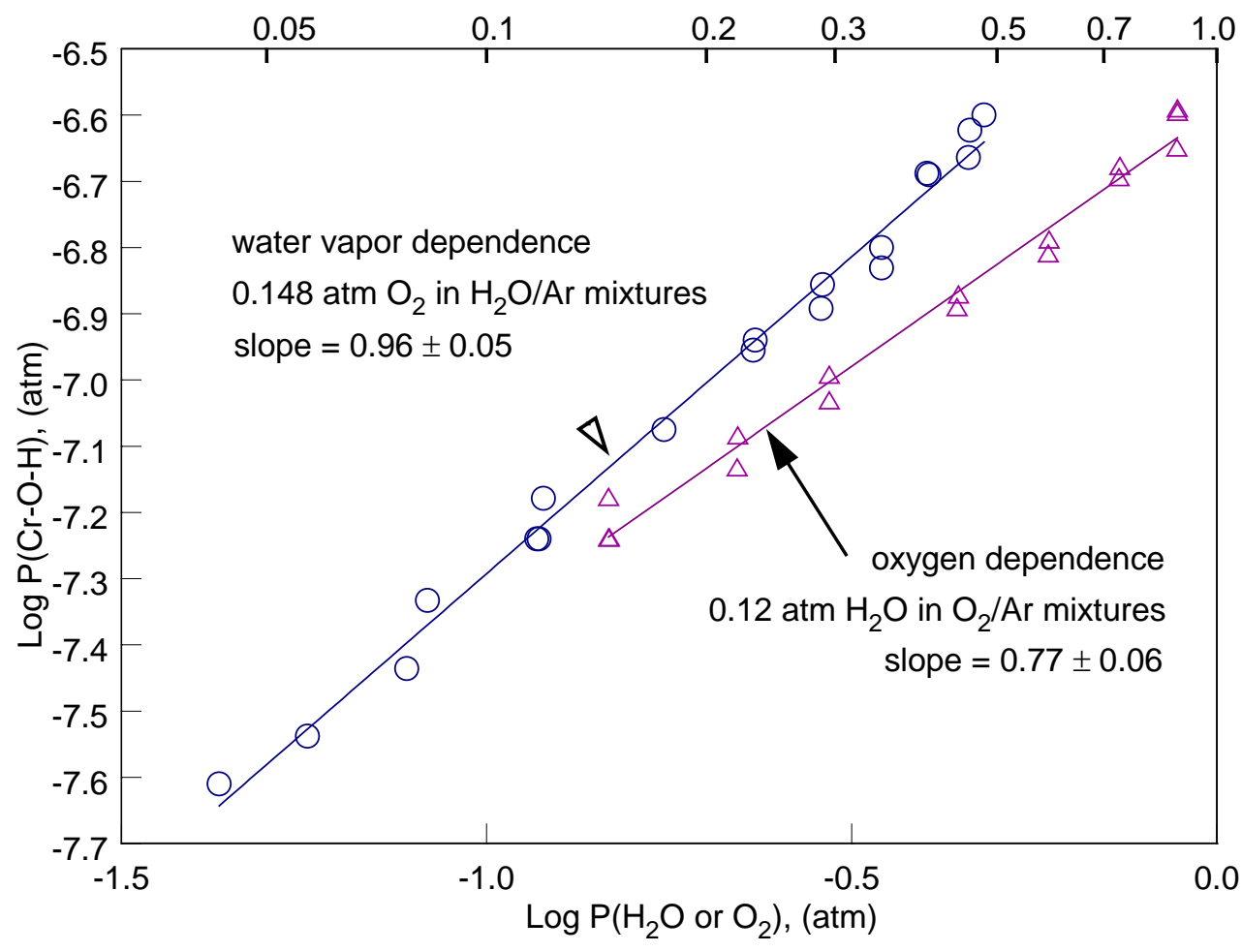

Opila et al, J. Phys. Chem. A. 111, 1971 (2007). 


\section{Temperature Dependence: $\mathrm{CrO}_{2}(\mathrm{OH})_{2}(\mathrm{~g})$ formation}

Temperature $(\mathrm{K})$

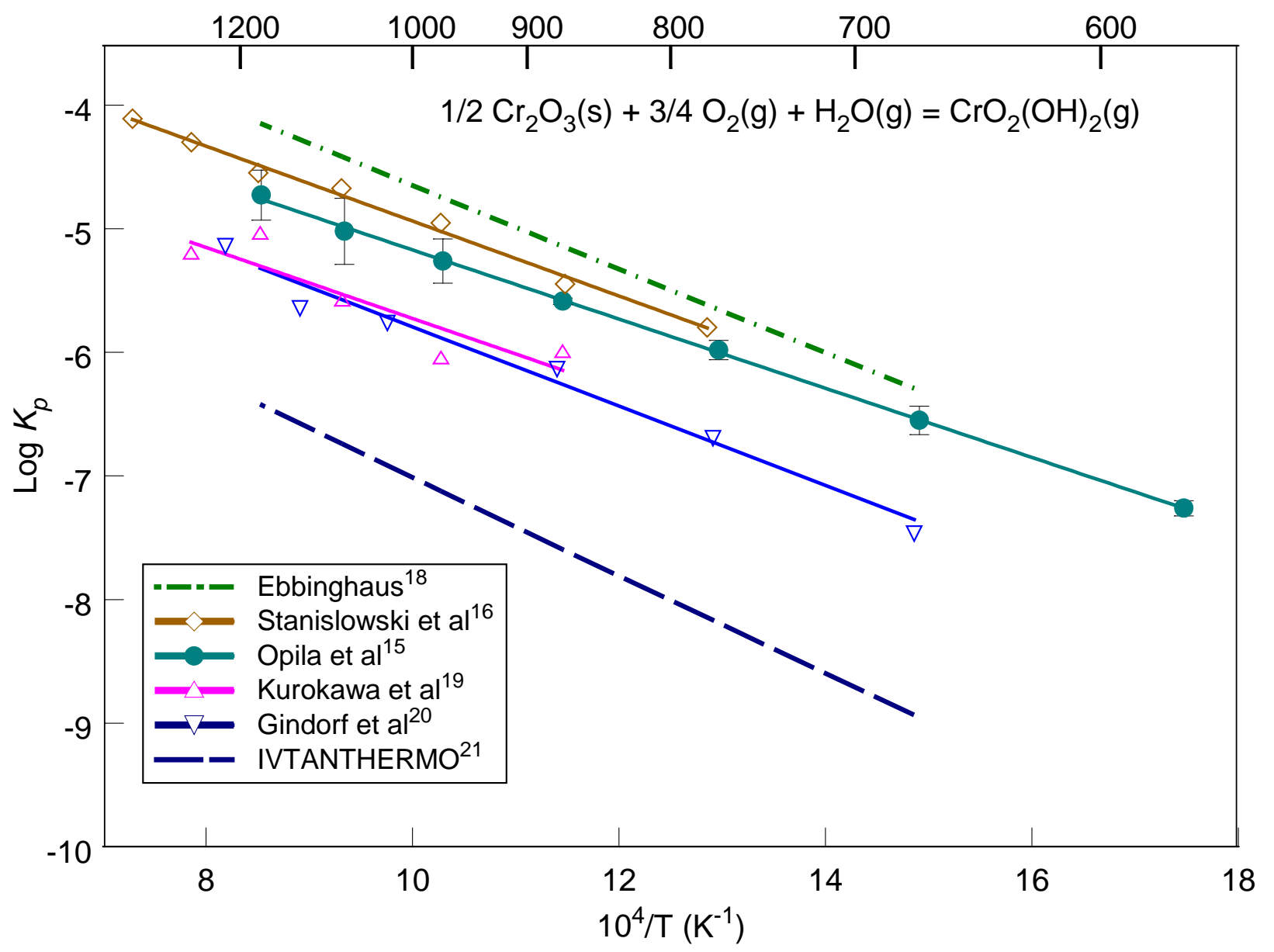

- Recent transpiration studies by Opila et al and Stanislowski et al have resolved discrepancy in thermochemical data for $\mathrm{CrO}_{2}(\mathrm{OH})_{2}(\mathrm{~g})$ formation

- Data of Opila et al: $\Delta H^{\circ}{ }_{r, 861 \mathrm{~K}}=53.5 \mathrm{~kJ} / \mathrm{mol}, \Delta{S^{\circ}}_{\mathrm{r}, 861 \mathrm{~K}}=-45.6 \mathrm{~J} / \mathrm{K} \mathrm{mol}$ 


\section{The Si-O-H system}

- $\mathrm{SiO}_{2}$ in SOFC: constituent of sealing glasses

- Possible Si-O-H(g) vapor species and experimental method used for identification

$$
\begin{aligned}
& \mathrm{SiO}_{2}(\mathrm{~s})+2 \mathrm{H}_{2} \mathrm{O}(\mathrm{g})=\mathrm{Si}(\mathrm{OH})_{4}(\mathrm{~g}) \\
& \mathrm{SiO}_{2}(\mathrm{~s})+\mathrm{H}_{2} \mathrm{O}(\mathrm{g})=\mathrm{SiO}(\mathrm{OH})_{2}(\mathrm{~g}) \\
& \mathrm{SiO}_{2}(\mathrm{~s})+1 / 2 \mathrm{H}_{2} \mathrm{O}(\mathrm{g})=\mathrm{SiO}(\mathrm{OH})(\mathrm{g})+1 / 4 \mathrm{O}_{2}(\mathrm{~g})
\end{aligned}
$$

transpiration $^{1,2}$, HPMS $^{3}$

KEMS $^{4}$

$\mathrm{KEMS}^{4}$

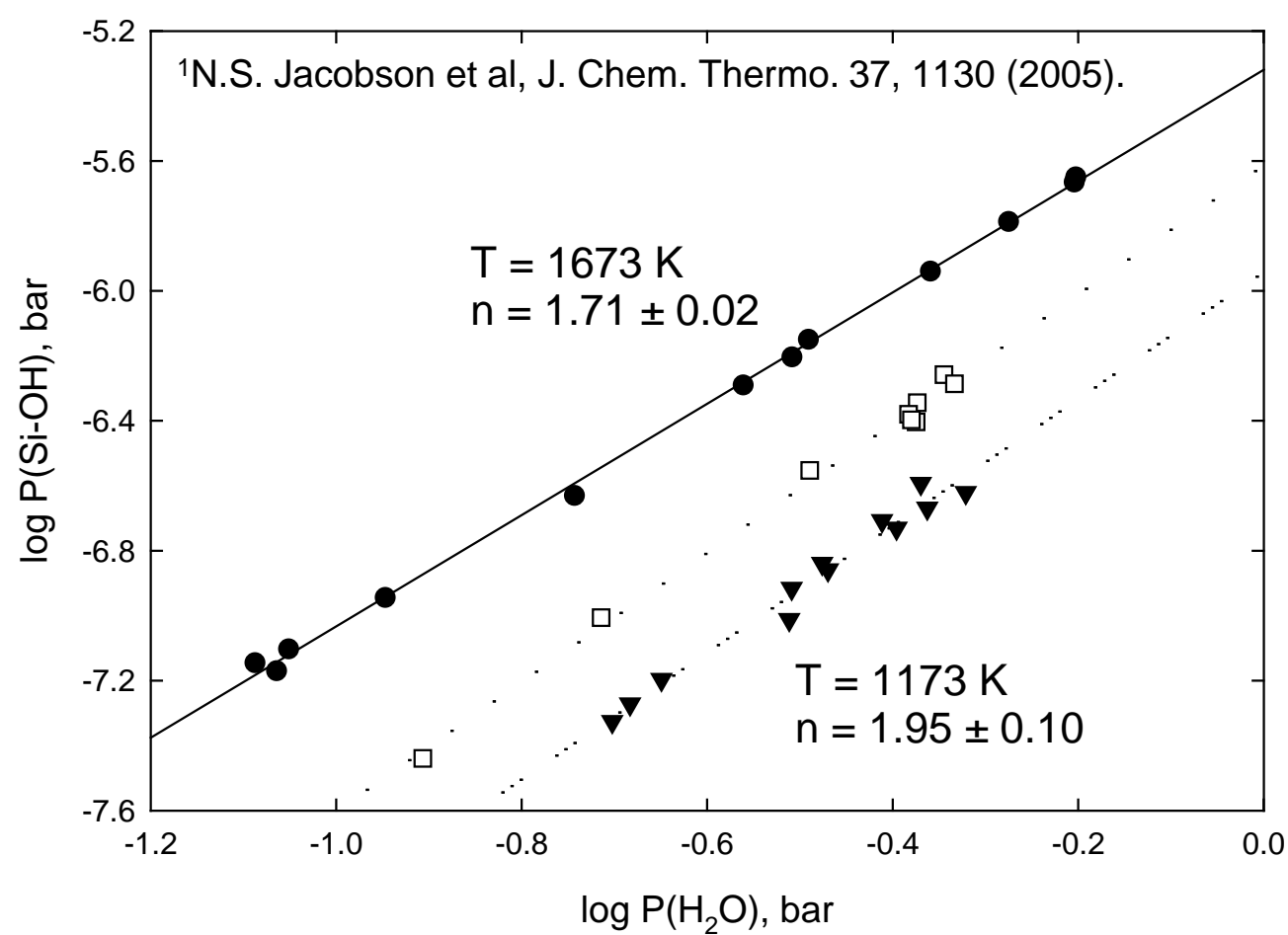

$\mathrm{T}=1373 \mathrm{~K}$

$\mathrm{n}=1.99 \pm 0.06$

Transpiration studies show pressure dependence consistent with $\mathrm{Si}(\mathrm{OH})_{4}(\mathrm{~g})$ formation

${ }^{2}$ Hashimoto, Geochim. Cosmo. Acta 56, 511-532 (1992)

${ }^{3}$ Opila et al, J. Am. Ceram. Soc. 80 [4] 1009 (1997).

${ }^{4}$ Hildenbrand \& Lau, J. Chem. Phys., 101 [7] 6076 (1994). 


\section{The Si-O-H system}

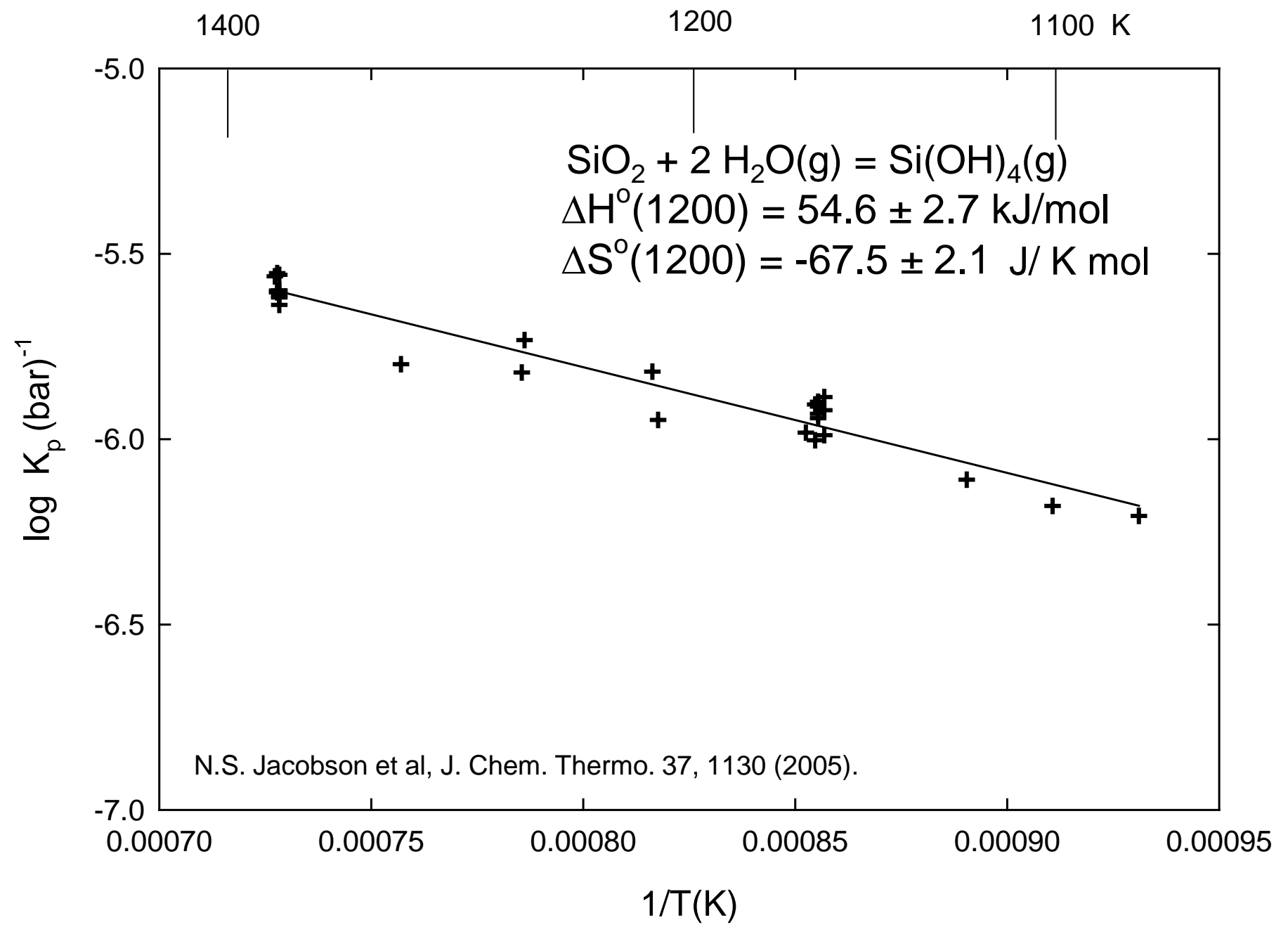

Temperature dependence for $\mathrm{Si}(\mathrm{OH})_{4}(\mathrm{~g})$ formation is relatively weak. 
- $\mathrm{Al}_{2} \mathrm{O}_{3}$ in SOFC: constituent of sealing glasses, insulators, and/or gas delivery components

- $\mathrm{Al}-\mathrm{O}-\mathrm{H}(\mathrm{g})$ vapor species identified from pressure dependence of $\mathrm{Al}_{2} \mathrm{O}_{3}$ volatility in TGA experiments and transpiration experiments with calcium aluminate

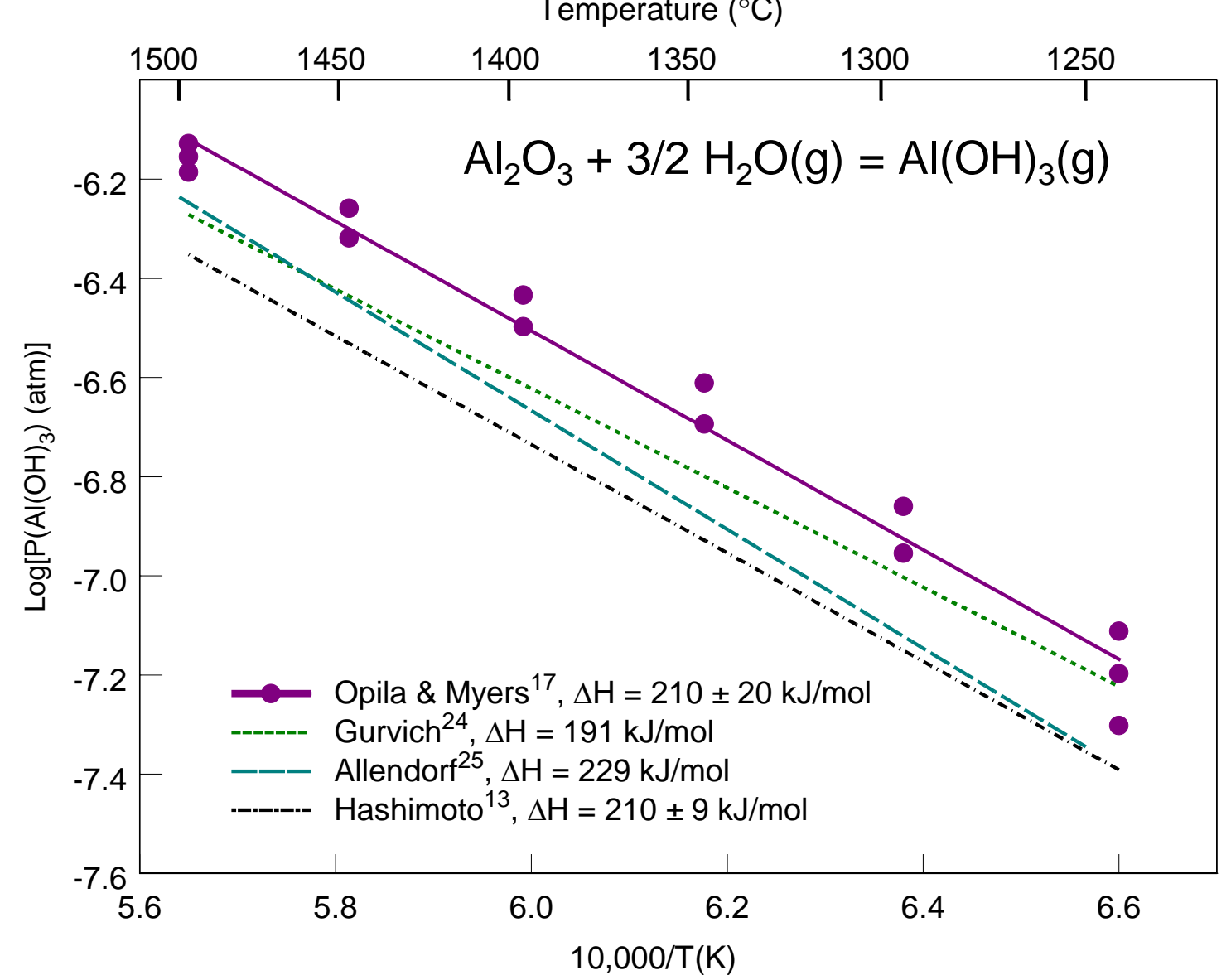




\section{Oxide volatility comparison}

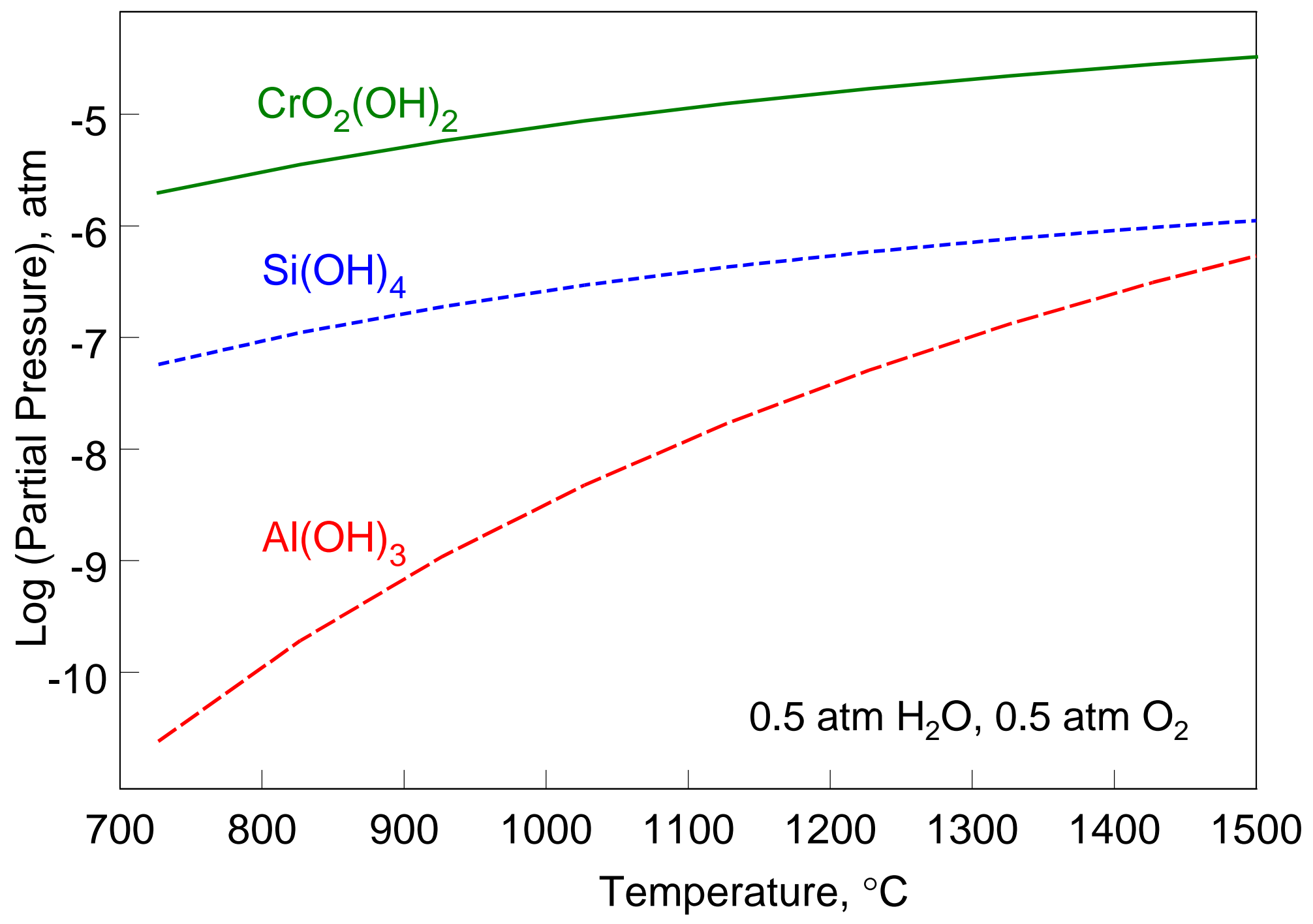




\section{Review of thermodynamic data for materials important in SOFC systems}

- Data base and literature review for $\mathrm{M}-\mathrm{O}-\mathrm{H}(\mathrm{g})$ data

- Data evaluation

- Availability of data for $\mathrm{M}-\mathrm{O}-\mathrm{H}(\mathrm{g})$

- Method by which data were obtained

- Completeness of data

- Reliability of data

- Calculation of $\mathrm{Ni}, \mathrm{CoO}, \mathrm{SrO}, \mathrm{CaO}, \mathrm{La}_{2} \mathrm{O}_{3}, \mathrm{MnO}, \mathrm{Pd}$, Pt stability in model anode/cathode environments using FactSage free energy minimization program

- Model anode environment: 1 bar $50 \% \mathrm{H}_{2} \mathrm{O}(\mathrm{g}) / 50 \% \mathrm{H}_{2}(\mathrm{~g})$

- Model cathode environment: 1 bar $50 \% \mathrm{H}_{2} \mathrm{O}(\mathrm{g}) / 50 \% \mathrm{O}_{2}(\mathrm{~g})$

- Ranking/summary of volatility trends 


\section{$\mathrm{Ni}-\mathrm{O}-\mathrm{H}$ and $\mathrm{Co}-\mathrm{O}-\mathrm{H}$ systems}

- $\quad \mathrm{Ni}$ used in Ni/YSZ anode, Co found in interconnect alloys and $\mathrm{CoO}$ in coatings

- $\quad \mathrm{Ni}-\mathrm{O}-\mathrm{H}$ vapor species include $\mathrm{Ni}(\mathrm{OH})_{2}, \mathrm{Ni}(\mathrm{OH}), \mathrm{NiH}, \mathrm{Ni}_{2}, \mathrm{NiO}$, and $\mathrm{Ni}$

- $\quad \mathrm{Ni}-\mathrm{O}-\mathrm{H}$ and Co-O-H systems studied by transpiration method in $\mathrm{H}_{2}, \mathrm{H}_{2} \mathrm{O}$

- G.R. Belton, A.S. Jordan, J. Phys. Chem. 71 [12] 4114 (1967).

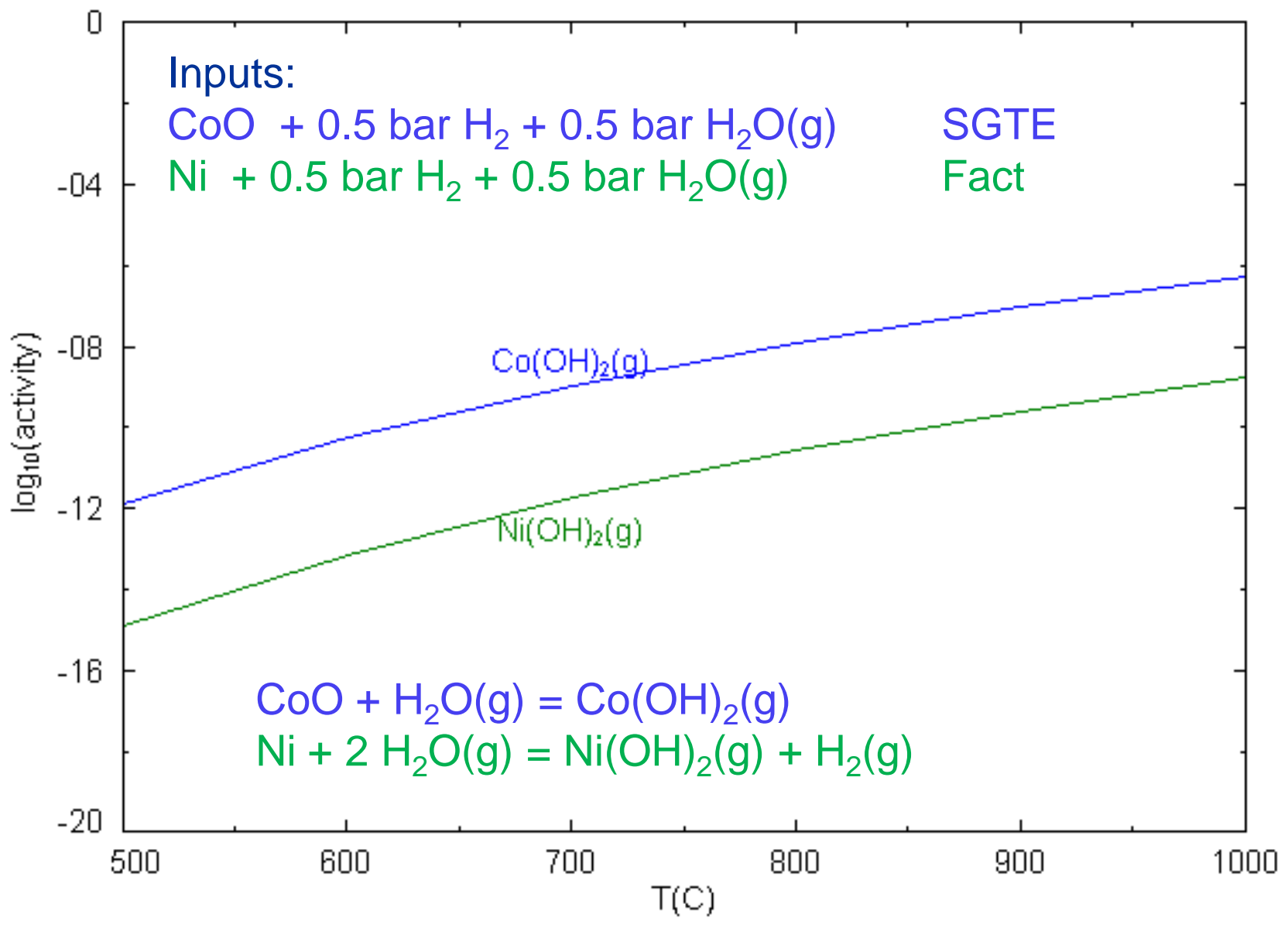




\section{Sr-O-H and Ca-O-H systems}

- $\quad \mathrm{SrO}$ found in LSM cathodes, $\mathrm{CaO}$ found in $\mathrm{La}_{1-\mathrm{x}} \mathrm{Ca}_{\mathrm{x}} \mathrm{CrO}_{3-\delta}$ interconnects

- $\quad \mathrm{Sr}-\mathrm{O}-\mathrm{H}$ vapor species include $\mathrm{Sr}(\mathrm{OH})_{2}, \mathrm{Sr}(\mathrm{OH}), \mathrm{SrH}, \mathrm{Sr}_{2}, \mathrm{SrO}, \mathrm{Sr}$, and $\mathrm{Sr}_{2} \mathrm{O}$

- $\mathrm{Ca}-\mathrm{O}-\mathrm{H}$ vapor species include $\mathrm{Ca}(\mathrm{OH})_{2}, \mathrm{Ca}(\mathrm{OH}), \mathrm{CaH}, \mathrm{Ca}_{2}, \mathrm{CaO}$, and $\mathrm{Ca}$

- Both systems studied by flame spectroscopy, $\mathrm{Ca}-\mathrm{O}-\mathrm{H}$ by transpiration

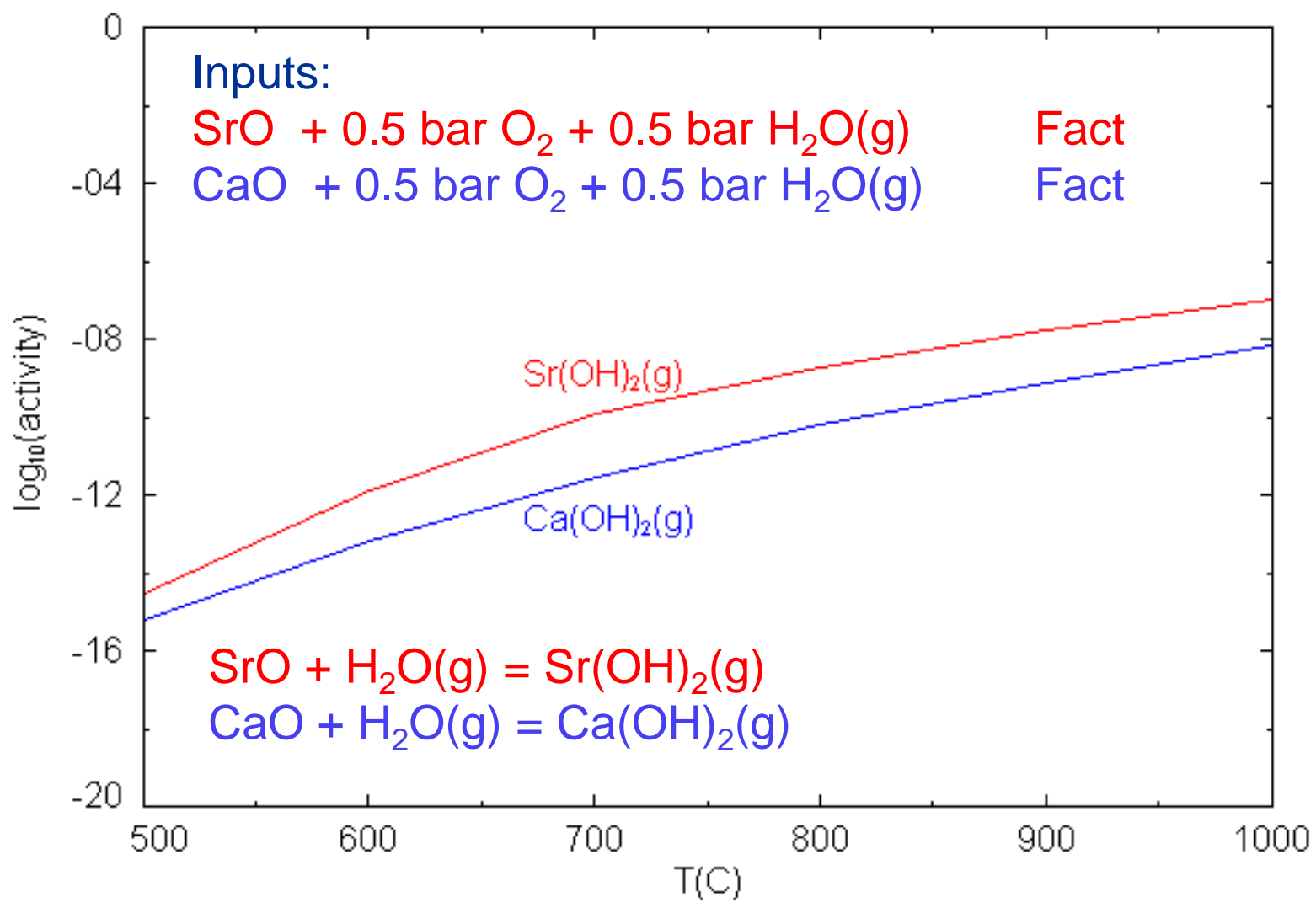




\section{Activity of components in complex oxides}

- $\mathrm{CaO}, \mathrm{Cr}_{2} \mathrm{O}_{3}$, and $\mathrm{La}_{2} \mathrm{O}_{3}$ activities, a(i), in $\mathrm{La}_{1-\mathrm{x}} \mathrm{Ca}_{\mathrm{x}} \mathrm{CrO}_{3-\delta}$ interconnects can be reduced relative to pure oxides.

- Volatility will be correspondingly reduced $\mathrm{Cr}_{2} \mathrm{O}_{3}+\mathrm{H}_{2} \mathrm{O}(\mathrm{g})+3 / 4 \mathrm{O}_{2}(\mathrm{~g})=\mathrm{CrO}_{2}(\mathrm{OH})_{2}(\mathrm{~g})$

$$
P_{\mathrm{CrO}_{2}(\mathrm{OH})_{2}}=K_{e q} a_{\mathrm{Cr}_{2} \mathrm{O}_{3}} P_{\mathrm{H}_{2} \mathrm{O}} P_{\mathrm{O}_{2}}^{3 / 4}
$$

- $\mathrm{CrO}_{2}(\mathrm{OH})_{2}(\mathrm{~g})$ formation from $\mathrm{La}_{1-x} \mathrm{Ca}_{\mathrm{x}} \mathrm{CrO}_{3-\delta}$ ( $x=0$ to 0.1 ) will be reduced by four orders of magnitude relative to pure $\mathrm{Cr}_{2} \mathrm{O}_{3}$

Thermodynamic activities of the components in $\mathrm{La}_{1-\mathrm{x}} \mathrm{Ca}_{\mathrm{x}} \mathrm{CrO}_{3-\mathrm{d}}, \mathrm{x}=0-0.21$ at $2000 \mathrm{~K}$.

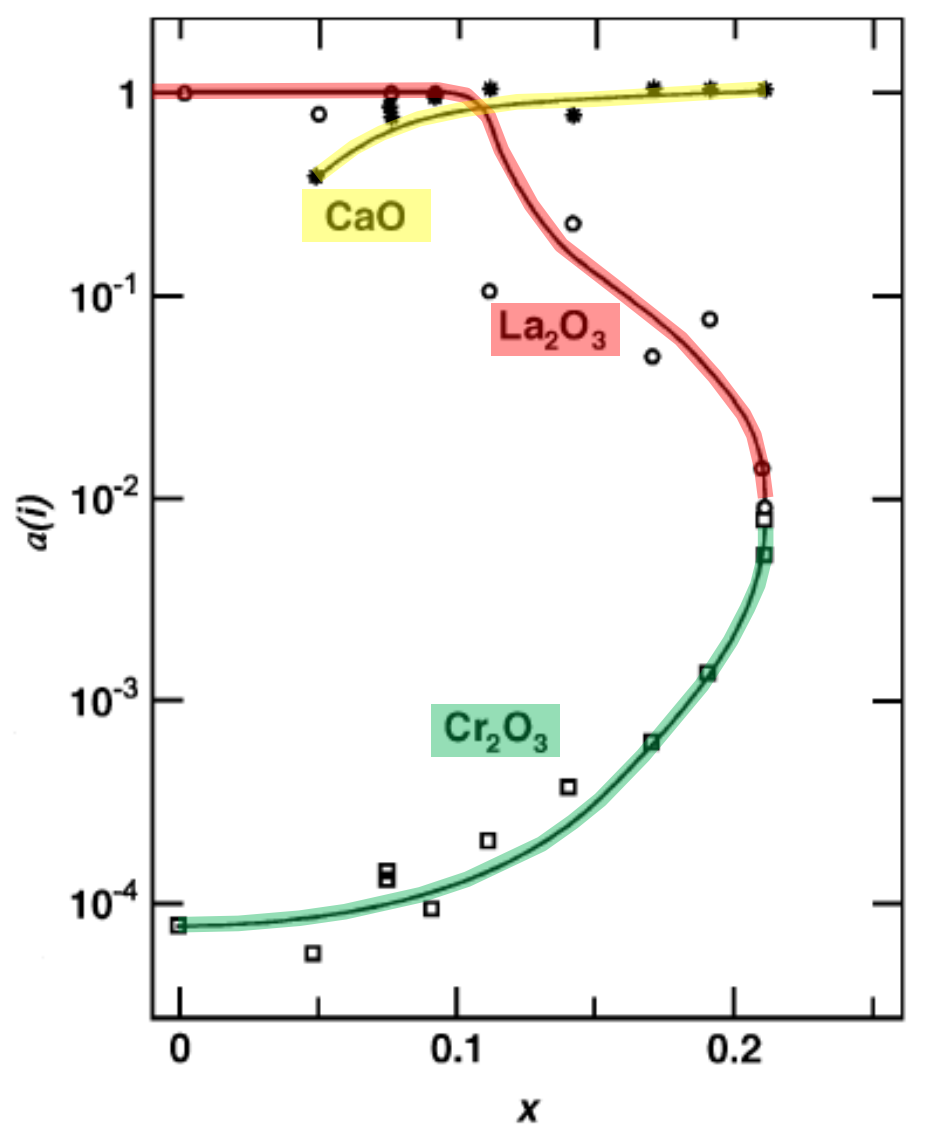

D.-H. Peck, M. Miller, K. Hilpert, Solid State Ionics 143, 391 (2001). 


\section{La-O-H system}

- $\mathrm{La}_{2} \mathrm{O}_{3}$ is a component of LSM cathodes and $\mathrm{La}_{1-\mathrm{x}} \mathrm{Ca}_{\mathrm{x}} \mathrm{CrO}_{3-\delta}$ interconnects

- La-Q-H vapor species include $\mathrm{La}(\mathrm{OH})_{3}, \mathrm{La}(\mathrm{OH})_{2}, \mathrm{La}(\mathrm{OH}), \mathrm{La}_{2}, \mathrm{LaO}, \mathrm{La}, \mathrm{La}_{2} \mathrm{O}$, $\mathrm{La}$, and $\mathrm{La}_{2} \mathrm{O}_{2}$

- Good review of La-O(g) system M. Heyrman, C. Chatillon, A. Pisch, Computer Coupling of Phase Diagrams \& Thermochemistry 28, 49 (2004).

- Origin of data for $\mathrm{La}(\mathrm{OH})_{3}(\mathrm{~g})$ unknown; $\mathrm{La}(\mathrm{OH})_{2}(\mathrm{~g})$ and $\mathrm{La}(\mathrm{OH})(\mathrm{g})$ data estimated

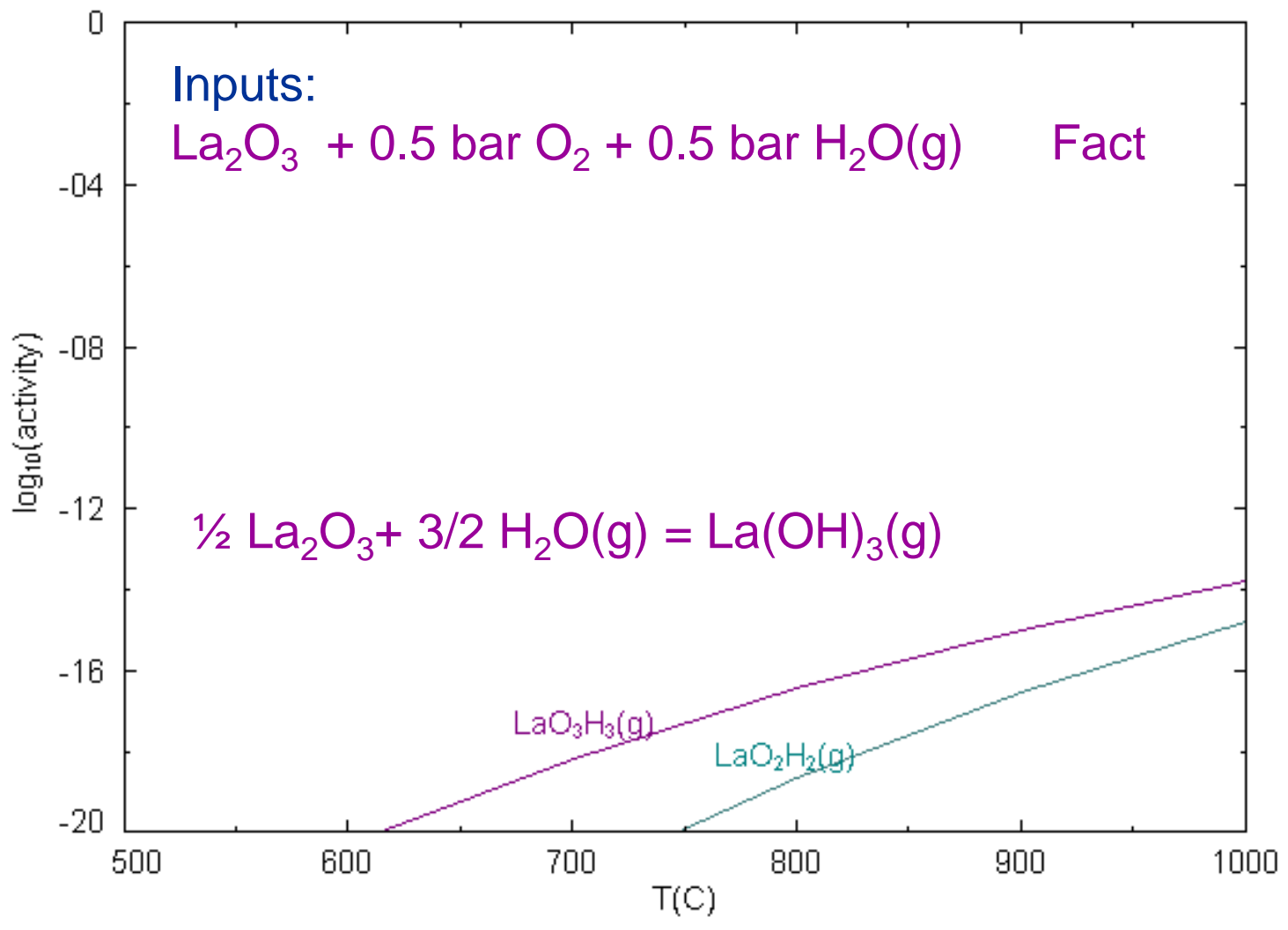




\section{Mn-O-H system}

- $\mathrm{MnO}$ is a component of LSM cathodes and oxide spinels formed/coated on metallic interconnects

- $\mathrm{Mn}-\mathrm{O}-\mathrm{H}$ vapor species include $\mathrm{Mn}(\mathrm{OH})_{2}, \mathrm{MnO}(\mathrm{OH}), \mathrm{Mn}(\mathrm{OH}), \mathrm{MnH}, \mathrm{MnO}, \mathrm{Mn}$, and $\mathrm{MnO}_{2}$

- $\mathrm{Mn}(\mathrm{OH})_{2}$ observed by KEMS; existence of $\mathrm{Mn}(\mathrm{OH})_{3}$ and $\mathrm{MnO}(\mathrm{OH})_{2}$ at higher pressures hypothesized: D.L. Hildenbrand, K.H. Lau, J. Chem. Phys. 100 [11] 8377 (1994).

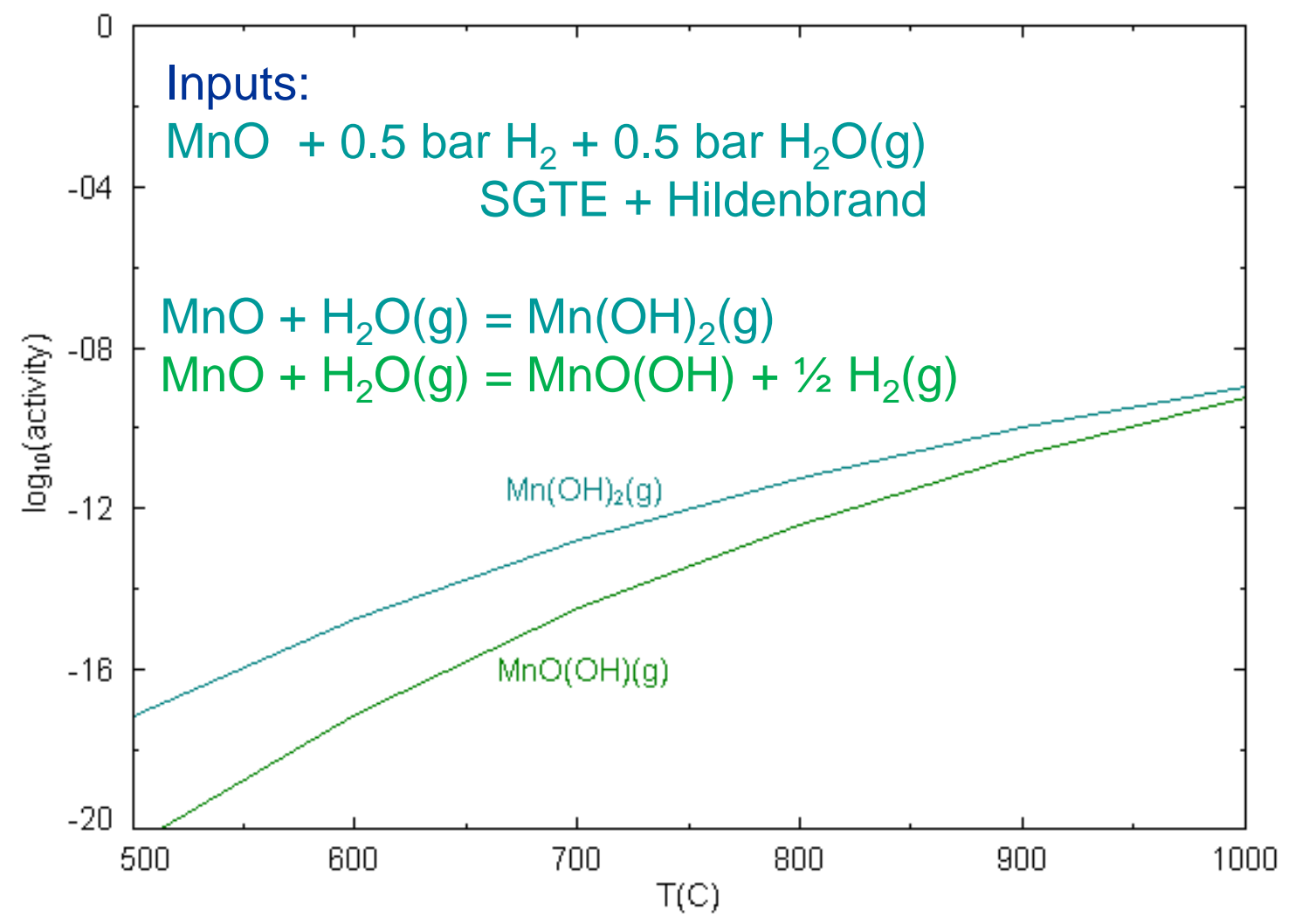




\section{Pd-O-H system}

- Pd used as current collector

- Pd-O-H vapor species include $\mathrm{PdOH}, \mathrm{Pd}, \mathrm{Pd}(\mathrm{OH})_{2}, \mathrm{PdO}$, and $\mathrm{PdO}_{2}$

- No experimental data for hydroxide vapor species, data are estimated

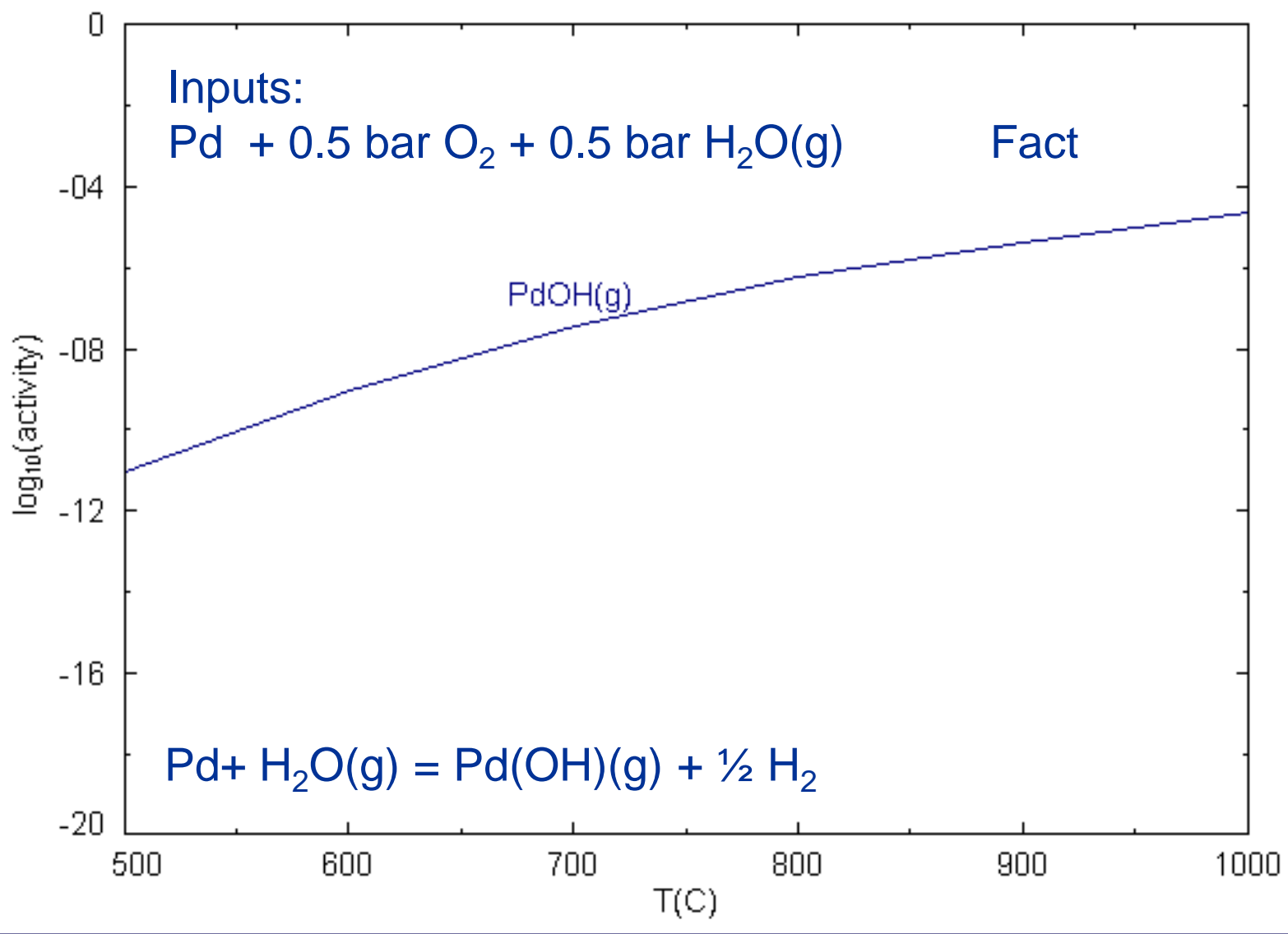




\section{Pt-O-H system}

- Pt used as current collector

- Pt-O-H vapor species include $\mathrm{PtO}_{2}, \mathrm{PtO}$, and Pt

- No data available for any hydroxide vapor species

- Experimental TGA results indicate that water vapor-Pt interactions are minimal

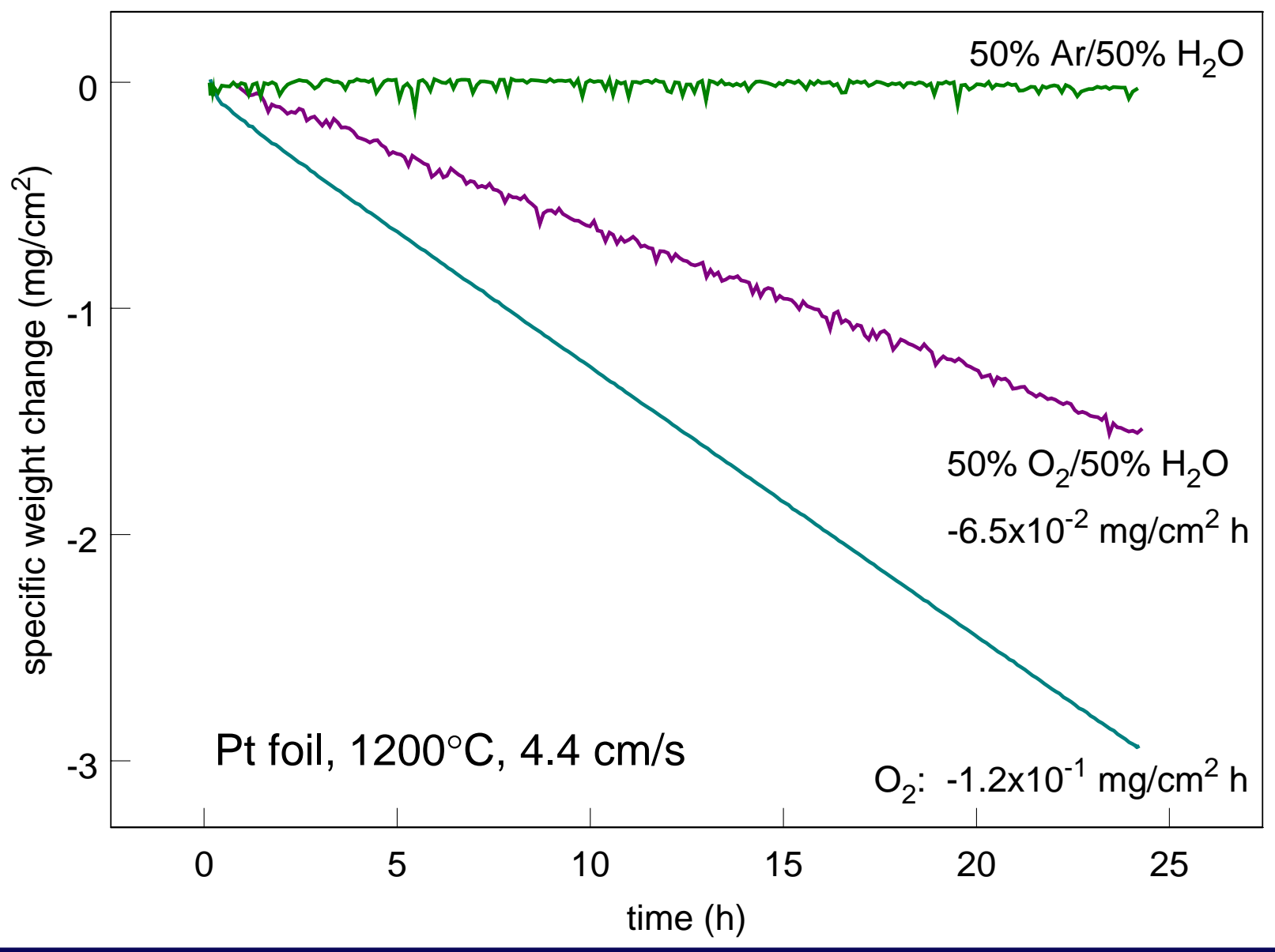




\section{Summary of equilibrium gaseous metal hydroxide partial pressures}

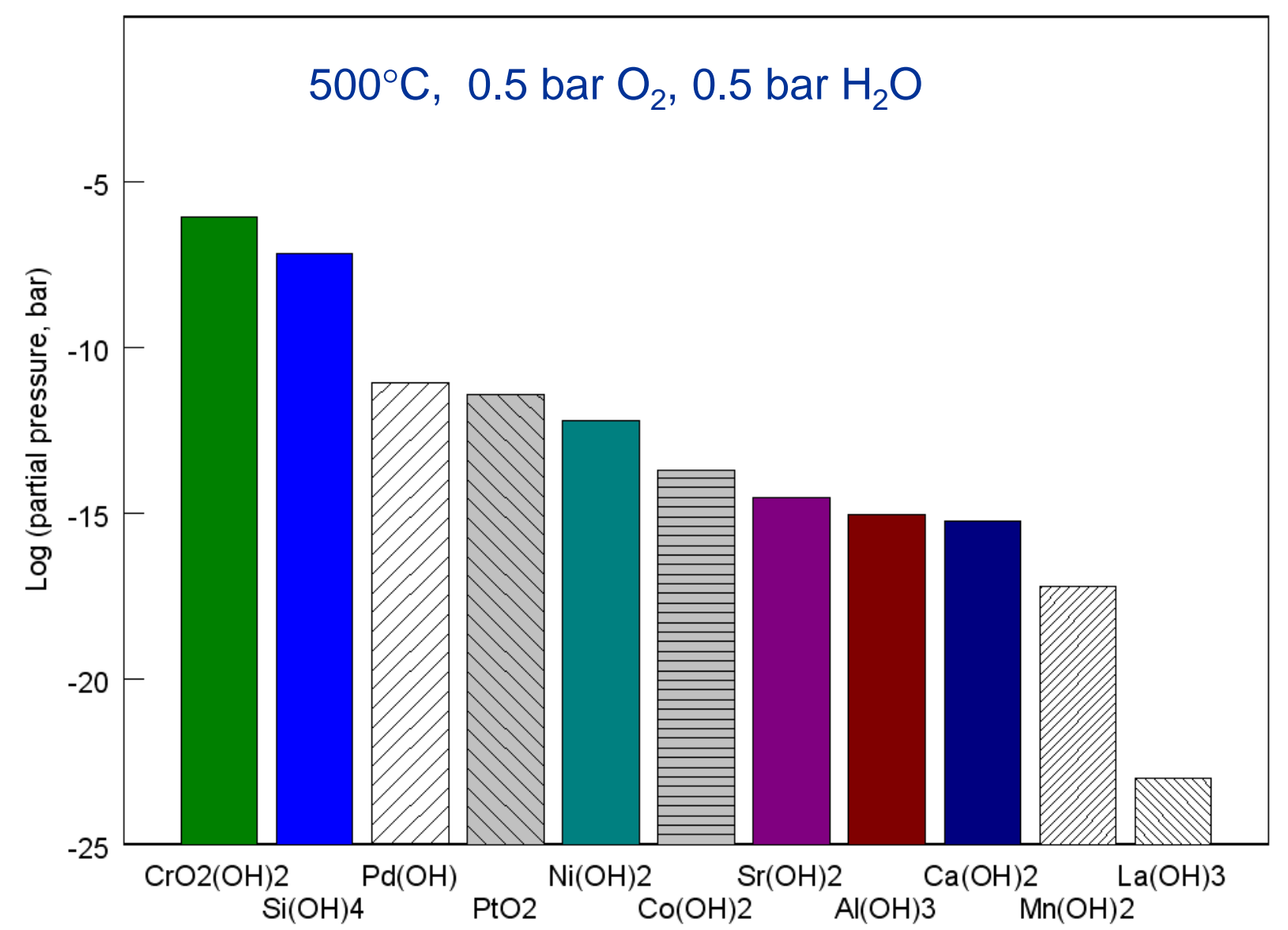

- Solid bars: good thermodynamic data

- Cross-hatched bars: thermodynamic data unreliable

- Gray cross-hatched bars: some experimental information about stability of gaseous metal hydroxides available, i.e $\mathrm{Co}(\mathrm{OH})_{2}(\mathrm{~g}), \mathrm{PtO}_{2}(\mathrm{~g})$ 


\section{Summary of equilibrium gaseous metal hydroxide} partial pressures: temperature effects

$500^{\circ} \mathrm{C}, 0.5$ bar $\mathrm{O}_{2}, 0.5$ bar $\mathrm{H}_{2} \mathrm{O}$
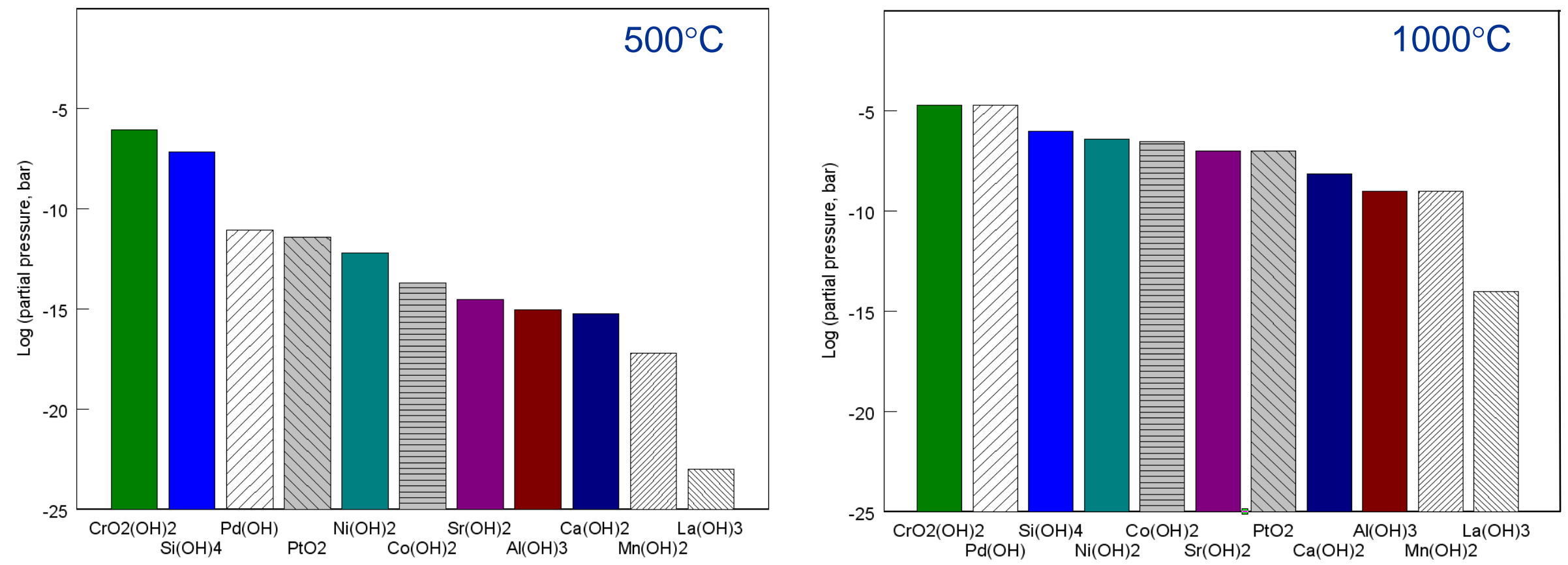

- Weak temperature dependence for $\mathrm{CrO}_{2}(\mathrm{OH})_{2}(\mathrm{~g})$ and $\mathrm{Si}(\mathrm{OH})_{4}(\mathrm{~g})$ : minimal durability benefit by reducing temperature

- Stronger temperature dependence for other gaseous metal hydroxides 
Summary of equilibrium gaseous metal hydroxide partial pressures: gas environment effects

$500^{\circ} \mathrm{C}$
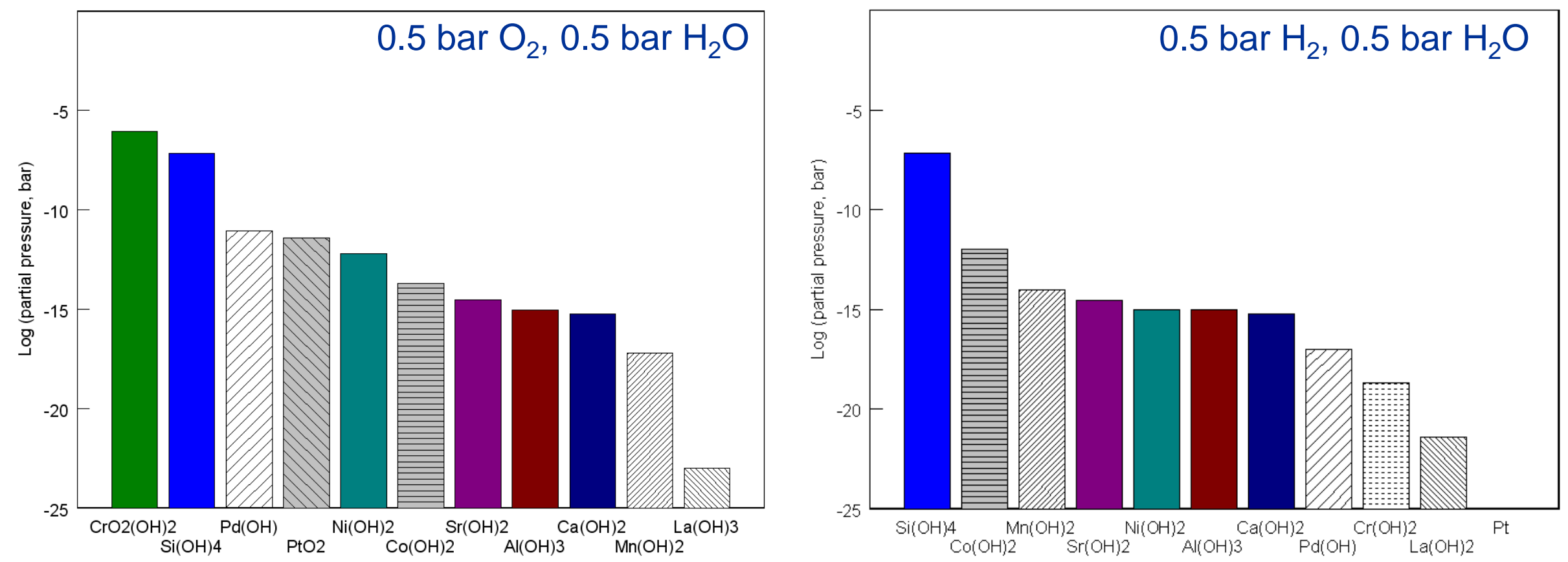

- $\mathrm{P}(\mathrm{MOH})$ dependent only on $\mathrm{P}\left(\mathrm{H}_{2} \mathrm{O}\right)$ do not change: $\mathrm{Si}(\mathrm{OH})_{4}(\mathrm{~g})$

- $\mathrm{P}(\mathrm{MOH})$ dependent on $\mathrm{P}\left(\mathrm{O}_{2}\right)$ and/or $\mathrm{P}\left(\mathrm{H}_{2}\right)$ can change dramatically: $\mathrm{CrO}_{2}(\mathrm{OH})_{2}(\mathrm{~g}) \rightarrow \mathrm{Cr}(\mathrm{OH})_{2}(\mathrm{~g})$

- $\mathrm{MO}$ - condensed phase reaction with environment can affect $\mathrm{P}(\mathrm{MOH})$ : $\mathrm{Ni} / \mathrm{NiO}$ or $\mathrm{Co} / \mathrm{CoO} / \mathrm{Co}_{3} \mathrm{O}_{4}$ 


\section{Kinetics of volatilization limited by transport through} laminar gaseous boundary layer

\section{$\mathrm{Si}(\mathrm{OH})_{4}(\mathrm{~g})$}

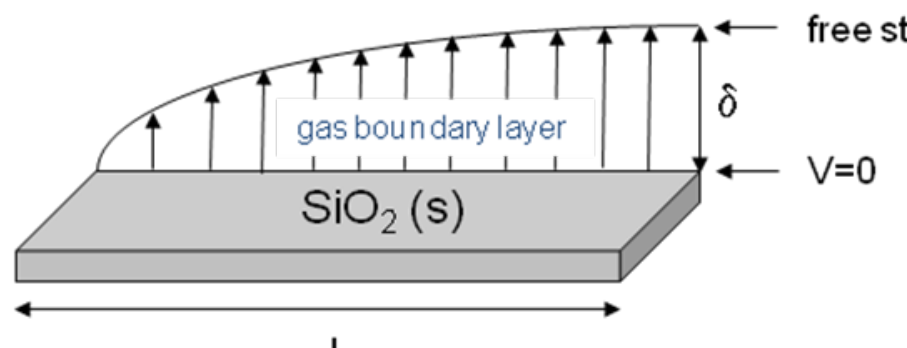

$$
J=0.664\left(\frac{\rho^{\prime} V L}{\eta}\right)^{1 / 2}\left(\frac{\eta}{\rho^{\prime} D}\right)^{1 / 3} \frac{D \rho}{L}
$$

Applications to SOFC

- Assumes laminar flow

- Assumes flat plate geometry - relevant for planar cell

- Related expression needed for mass transport from tube wall in flowing gas for tubular SOFC components

- More complete model needed to account for gas transport in pores of active surfaces
$\mathrm{J}=$ mass loss rate

$\rho^{\prime}=$ boundary layer gas density $\propto P_{\text {total }}$

$D=$ interdiffusion coefficient $\propto 1 / P_{\text {total }}$

$\rho=\mathrm{Si}(\mathrm{OH})_{4}$ gas density $\propto \mathrm{P}_{\mathrm{SiOH} 4}$

$v=$ linear gas velocity

$\eta=$ gas viscosity

$\mathrm{L}=$ characteristic length 


\section{Summary and conclusions}

- Experimental techniques are available for characterizing volatility of materials under conditions meaningful for SOFC applications

- Thermodynamic data for prediction of materials durability can be obtained

- Accurate thermodynamic data for $\mathrm{Cr}_{2} \mathrm{O}_{3}, \mathrm{SiO}_{2}, \mathrm{Al}_{2} \mathrm{O}_{3}, \mathrm{Ni}, \mathrm{SrO}$, $\mathrm{CaO}$ volatilization in high temperature water vapor are available

- Additional thermodynamic data are needed for gaseous hydroxides formed from $\mathrm{La}_{2} \mathrm{O}_{3}, \mathrm{MnO}, \mathrm{Pd}$, others?

- Component activities in complex oxides/alloys are needed to accurately predict materials durability in SOFC

- Kinetic models that accurately describe vapor transport in SOFC structures and incorporate gaseous metal hydroxide thermodynamics are needed 


\section{Acknowledgments}

The thermodynamic data evaluation was made possible, in part, through funding from Rolls-Royce Fuel Cell Systems, Inc. under NASA Space Act Agreement SAA3-1031. This material is based upon work supported by the Department of Energy National Energy Technology Laboratory under Award Number DE-FE0000303." 


\section{Fe-O-H system}

- $\quad$ Fe found in interconnect alloys and LSF cathodes

- $\mathrm{Fe}-\mathrm{O}-\mathrm{H}$ vapor species include $\mathrm{Fe}(\mathrm{OH})_{2}, \mathrm{Fe}(\mathrm{OH}), \mathrm{FeO}_{2}, \mathrm{FeO}$, and $\mathrm{Fe}$

- Fe-O-H system studied by transpiration method in $\mathrm{H}_{2}, \mathrm{H}_{2} \mathrm{O}$

- G.R. Belton, F.D. Richardson, Trans. Faraday Soc. 58,1562 (1962).

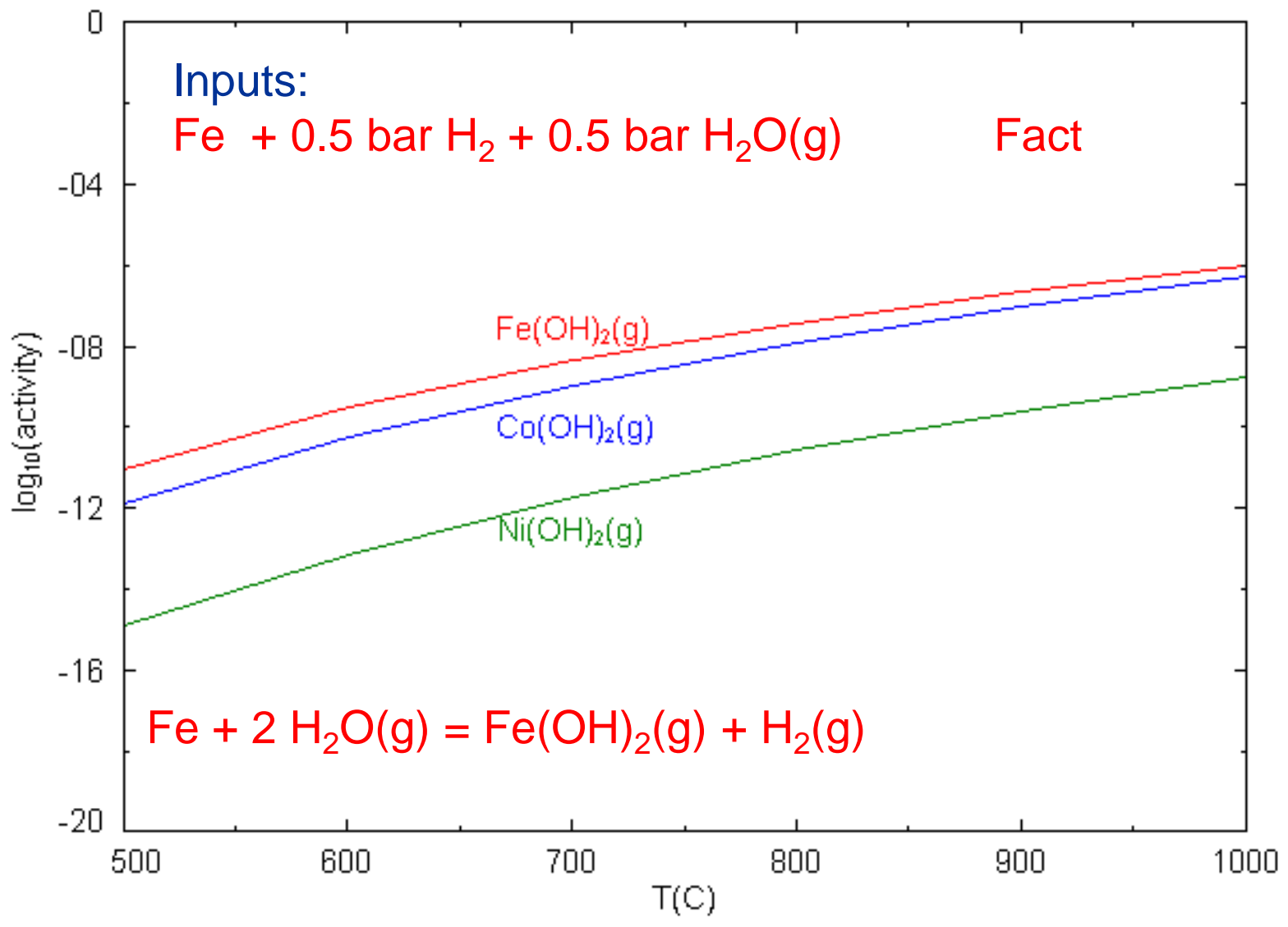

